\title{
New Chiral Synthons for Efficient Preparation of Bispropionates via Stereospecific Oxonia-Cope Rearrangements
}

\author{
Yi-Hung Chen and Frank E. McDonald* \\ Department of Chemistry, Emory University, Atlanta, GA 30322
}

\section{Supporting Information - part I}

\section{Table of Contents}

General experimental information

S-2

Preparation of allylic benzoate 2

Preparation of racemic bispropionate synthons $\mathbf{3}, \mathbf{4}$ (Scheme 1)

S-4

Kinetic Resolution of bispropionate synthon 3

S-5

Kinetic Resolution of bispropionate synthon 4

S-6

Basic methanolysis of 5 and $\mathbf{6}$

S-7

Bispropionate transfer to form: $\mathbf{7 b}$ (Scheme 2)

S-8

$7 \mathrm{a}$

S-9

10a

S-9

$10 b$

S-10

13 (Table 1)

Preparation of (-)-Invictolide 21 (Scheme 3)

For ${ }^{1} \mathrm{H}$ and ${ }^{13} \mathrm{C}$ NMR spectra, see part II (S-21 through S-49) 
General information: ${ }^{1} \mathrm{H}$ NMR and ${ }^{13} \mathrm{C}$ NMR spectra were recorded on a Varian INOVA-400 spectrometer (400 MHz for ${ }^{1} \mathrm{H}, 100 \mathrm{MHz}$ for ${ }^{13} \mathrm{C}$ ), NMR spectra were recorded on solutions in deuterated chloroform $\left(\mathrm{CDCl}_{3}\right)$ with residual chloroform $\left(\delta 7.26 \mathrm{ppm}\right.$ for ${ }^{1} \mathrm{H}$ NMR and $\delta 77.0$ ppm for $\left.{ }^{13} \mathrm{C} N M R\right)$, or deuterated benzene $\left(\mathrm{C}_{6} \mathrm{D}_{6}\right)$ with residual benzene $\left(\delta 7.16 \mathrm{ppm}\right.$ for ${ }^{1} \mathrm{H}$ NMR and $\delta 128.4 \mathrm{ppm}$ for ${ }^{13} \mathrm{C} \mathrm{NMR}$ ) taken as the standard, and were reported in parts per million (ppm). Abbreviations for signal coupling are as follows: s, singlet; d, doublet; $t$, triplet; $q$, quartet; m, multiplet. IR spectra were collected on a Mattson Genesis II FT-IR spectrometer as neat films. Mass spectra (high resolution APCI) was recorded on a Finnigan LTQ-FTMS Mass spectrometer. High performance liquid chromatography (HPLC) was performed on HewlettPackard 1100 Series chromatography using Chiralpak AS-RH $(15 \mathrm{~cm})$ and Chiralpak AS $(25 \mathrm{~cm})$ columns. Elemental analyses were performed by Atlantic Microlab Inc, P. O. Box 2288, Norcross, Georgia. Optical rotations were measured using a Perkin-Elmer 341 polarimeter. Analytical Thin Layer Chromatography (TLC) was performed on precoated glass backed plates purchased from Whatman (silica gel 60F254; 0.25mm thickness). Flash column chromatography was carried out with silica gel 60 (230-400 mesh ASTM) from EM Science. All reactions were carried out with anhydrous solvents in oven-dried or flame-dried and argon-charged glassware. All anhydrous solvents except as mentioned were dried with $4 \AA$ molecular sieves (beads) purchased from Sigma-Aldrich and tested for trace water content with Coulometric KF Titrator from Denver Instruments. All solvents used in extraction procedures and chromatography were used as received from commercial suppliers without prior purification. All reagents were purchased from Sigma-Aldrich or Strem Chemicals. 


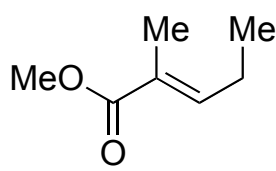

$\underset{\text { (2) } \mathrm{NaOBz}}{\stackrel{\mathrm{NBS}, \mathrm{h} v / \Delta}{\longrightarrow}}$

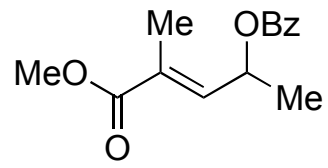

2

2-methyl-4-(benzoyloxy)-pent-2-enoic acid methyl ester (2). In a $250 \mathrm{~mL}$ round-bottom flask with reflux condenser, $N$-bromosuccinimide (NBS, $9.18 \mathrm{~g}, 51.15 \mathrm{mmol}$ ) was added to a solution of methyl trans-2-methyl-2-pentenoate $(6.02 \mathrm{~g}, 46.50 \mathrm{mmol})$ in $\mathrm{CCl}_{4}(70 \mathrm{~mL})$ at room temperature. The resulting solution was irradiated with a $150 \mathrm{~W}$ bulb for $3 \mathrm{~h}$, allowing the reaction mixture to boil to reflux temperature. ${ }^{1}$ After cooling, the reaction mixture was diluted with hexane $(50 \mathrm{~mL})$ and passed through a short plug of silica. The solvents were removed by rotary evaporation, and the crude mixture containing an allylic bromide was used for the next step, without further purification.

The allylic bromide intermediate was dissolved in DMF $(80 \mathrm{~mL})$, and sodium benzoate (9.02 g, $62.45 \mathrm{mmol})$ was added at room temperature. The resultant solution was stirred at 100 ${ }^{\circ} \mathrm{C}$ for $3 \mathrm{~h}$, cooled to room temperature and diluted with water $(200 \mathrm{~mL})$. The allylic benzoate was extracted with ethyl acetate and the organic layer was dried over $\mathrm{MgSO}_{4}$ followed by concentration to afford a yellowish oil. The residue was purified by flash chromatography on silica gel $($ EtOAc/Hexane $=1: 6)$ to yield compound 2 (7.62 g, 66\% for 2 steps). ${ }^{1} \mathrm{H}$ NMR (400 $\left.\mathrm{MHz}, \mathrm{CDCl}_{3}\right) \delta 8.05(\mathrm{dd}, 2 \mathrm{H}, J=8.2,1.2 \mathrm{~Hz}), 7.56(\mathrm{tt}, 1 \mathrm{H}, J=7.0,1.2 \mathrm{~Hz}), 7.44(\mathrm{dd}, 2 \mathrm{H}, J=$ 8.2, $7.0 \mathrm{~Hz}), 6.75(\mathrm{dq}, 1 \mathrm{H}, J=8.4,1.4 \mathrm{~Hz}), 5.90-5.83(\mathrm{~m}, 1 \mathrm{H}), 3.75$ (s, 3H), 1.99 (d, 3H, $J=1.4$ $\mathrm{Hz}), 1.47(\mathrm{~d}, 3 \mathrm{H}, J=6.8 \mathrm{~Hz}) ;{ }^{13} \mathrm{C}\left(100 \mathrm{MHz}, \mathrm{CDCl}_{3}\right) \delta 168.1,165.8,139.9,133.0,130.1,129.6$, 129.3, 128.3, 68.2, 52.0, 19.7, 12.9; HRMS (APCI): $m / z$ calcd. for $\mathrm{C}_{14} \mathrm{H}_{17} \mathrm{O}_{4}\left(\mathrm{M}^{+}\right)$249.1127, found 249.1120; FT-IR (KBr) 2988, 2934, 2849, 1718, 1660, 1602, $1451 \mathrm{~cm}^{-1}$.

(1) Sydnes, L. K.; Skattebøl, L.; Chapleo, C. B.; Leppard, D. G.; Svanholt, K. L.; Dreiding, A. S. Helv. Chim. Acta 1975, 58, 2061. 

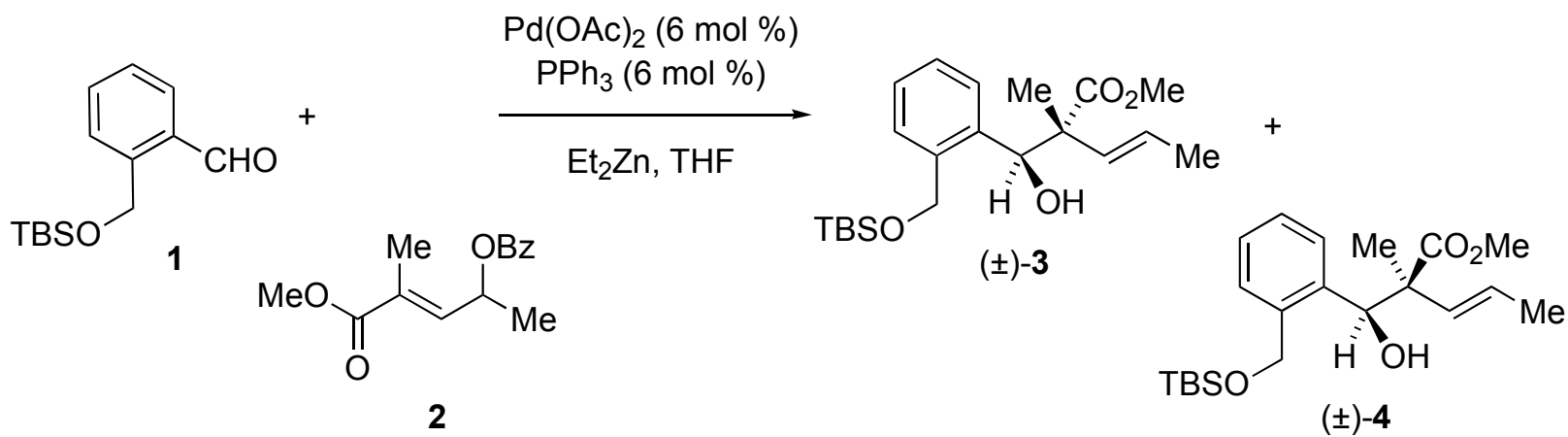

2-\{[2-(tert-Butyldimethylsilanyloxymethyl)-phenyl]-hydroxymethyl\}-2-methylpent-3-enoic acid methyl ester $(3,4) . \mathrm{Pd}(\mathrm{OAc})_{2}(61 \mathrm{mg}, 0.27 \mathrm{mmol})$ was dissolved in freshly distilled THF $(30 \mathrm{~mL})$ under argon, and cooled to $0{ }^{\circ} \mathrm{C}$. $\mathrm{PPh}_{3}(71 \mathrm{mg}, 0.27 \mathrm{mmol})$, a solution of aldehyde $1^{2}$ $(1.11 \mathrm{~g}, 4.43 \mathrm{mmol})$ and allylic benzoate $2(1.10 \mathrm{~g}, 4.43 \mathrm{mmol})$ in THF $(10 \mathrm{~mL})$ were added under argon followed by dropwise addition of diethylzinc $(22.15 \mathrm{~mL}, 1 \mathrm{M}$ in hexane, 22.15 mmol). The mixture was stirred at $0{ }^{\circ} \mathrm{C}$ for $5 \mathrm{~min}$ and room temperature for $20 \mathrm{~h}$. The reaction was quenched by dropwise addition of $5 \% \mathrm{HCl}$ at $0{ }^{\circ} \mathrm{C}$ (Caution: evolution of ethane gas) and diluted with ether. After the mixture was warmed to room temperature the layers were separated and aqueous layer was extracted with ether. The combined organic solution was washed with saturated brine and dried over $\mathrm{MgSO}_{4}$. The solvent was removed under reduced pressure and the residue was chromatographed on silica gel (Ether/Pentane $=1: 6$ with 1 2\% methanol) to afford homoallylic alcohol 3 (348 mg, $0.92 \mathrm{mmol}$ ) and the diastereomer 4 (984 mg, $2.60 \mathrm{mmol})$ in 80\% combined yield.

$( \pm)-3:{ }^{1} \mathrm{H}$ NMR $\left(400 \mathrm{MHz}, \mathrm{CDCl}_{3}\right) \delta 7.42(\mathrm{~d}, 1 \mathrm{H}, J=7.2 \mathrm{~Hz}), 7.36(\mathrm{dd}, 1 \mathrm{H}, J=7.6,1.6 \mathrm{~Hz})$, 7.29-7.20 (m, 2H), $5.90(\mathrm{dq}, 1 \mathrm{H}, J=16.0,1.8 \mathrm{~Hz}), 5.47-5.38(\mathrm{~m}, 1 \mathrm{H}), 5.21(\mathrm{~s}, 1 \mathrm{H}), 4.79(\mathrm{AB}$, 2H, $J=12.8 \mathrm{~Hz}), 3.73(\mathrm{~s}, 3 \mathrm{H}), 1.75(\mathrm{dd}, 3 \mathrm{H}, J=6.4,1.8 \mathrm{~Hz}), 1.17$ (s, 3H), 0.93 (s, 9H), 0.099 (s, $3 \mathrm{H}), 0.097(\mathrm{~s}, 3 \mathrm{H}) ;{ }^{13} \mathrm{C}\left(100 \mathrm{MHz}, \mathrm{CDCl}_{3}\right) \delta 177.1,138.9,137.0,129.4,128.1,127.7,127.3$, 127.2, 126.5, 73.9, 63.1, 53.5, 52.4, 25.9, 18.7 (two overlapping carbons), 18.3, -5.3; HRMS (APCI): $m / z$ calcd. for $\mathrm{C}_{21} \mathrm{H}_{35} \mathrm{O}_{4} \mathrm{Si}\left(\mathrm{M}^{+}\right)$379.2305, found 379.2299; FT-IR (KBr) 3475, 3034, 2953, 1724, 1462, $1253 \mathrm{~cm}^{-1}$; Anal. calcd. for $\mathrm{C}_{21} \mathrm{H}_{34} \mathrm{O}_{4} \mathrm{Si}: \mathrm{C}, 66.62 ; \mathrm{H}, 9.05$, found: $\mathrm{C}, 66.78$; $\mathrm{H}$, 9.10 .

$( \pm)-4:{ }^{1} \mathrm{H}$ NMR $\left(400 \mathrm{MHz}, \mathrm{CDCl}_{3}\right) \delta$ 7.36-7.31 (m, 2H), 7.19-7.15 (m, 2H), $5.34(\mathrm{dq}, 1 \mathrm{H}, J=$ 15.6, 1.4 Hz), 5.27-5.22 (m, 1H), $5.23(\mathrm{~s}, 1 \mathrm{H}), 4.64$ (AB, 2H, J=13.2 Hz), 3.67 (s, 3H), $1.52(\mathrm{dd}$,

(2) Anderson, W. K.; Kinder, F. R. J. Heterocycl. Chem. 1990, 27, 975. 
$3 \mathrm{H}, J=6.0,1.4 \mathrm{~Hz}), 1.17(\mathrm{~s}, 3 \mathrm{H}), 0.84(\mathrm{~s}, 9 \mathrm{H}), 0.00(\mathrm{~s}, 6 \mathrm{H}) ;{ }^{13} \mathrm{C}\left(100 \mathrm{MHz}, \mathrm{CDCl}_{3}\right) \delta 176.3$, 138.8, 137.0, 131.6, 127.6, 127.5, 127.0, 126.6, 126.4, 73.2, 62.9, 54.8, 52.2, 25.9, 18.3, 18.2, 16.0, -5.3; HRMS (APCI): $m / z$ calcd. for $\mathrm{C}_{21} \mathrm{H}_{35} \mathrm{O}_{4} \mathrm{Si}\left(\mathrm{M}^{+}\right)$379.2305, found 379.2299; FT-IR (KBr) 3497, 3034, 2953, 1725, 1459, $1254 \mathrm{~cm}^{-1}$; Anal. calcd. for $\mathrm{C}_{21} \mathrm{H}_{34} \mathrm{O}_{4} \mathrm{Si}$ : C, 66.62; $\mathrm{H}, 9.05$, found: $\mathrm{C}, 66.77 ; \mathrm{H}, 9.18$.

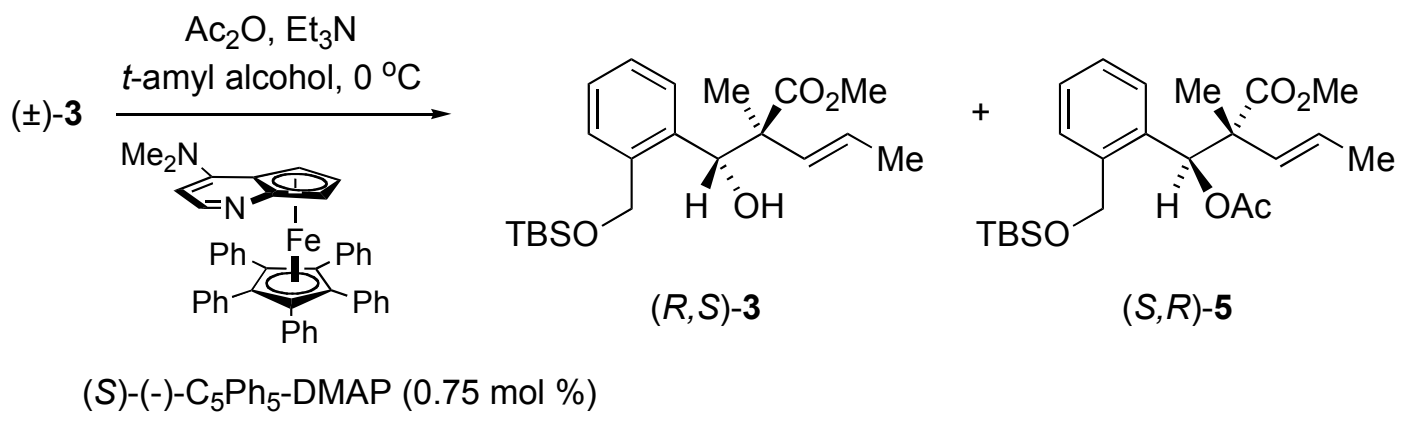

Kinetic resolution of 3. The racemic homoallylic alcohol $( \pm)-3(1.42 \mathrm{~g}, 3.75 \mathrm{mmol})$, catalyst $(S)-(-)-\mathrm{C}_{5} \mathrm{Ph}_{5}$-DMAP $(20 \mathrm{mg}, 0.03 \mathrm{mmol})$ and triethylamine $(0.39 \mathrm{~mL}, 2.81 \mathrm{mmol})$ in tert-amyl alcohol $(8.8 \mathrm{~mL})$ was gently heated to dissolve the catalyst. After the catalyst was completely dissolved, the purple solution was cooled in an ice bath and acetic anhydride $(0.27 \mathrm{~mL}, 2.81$ mmol) was added by syringe. The resulting solution was stirred at $0{ }^{\circ} \mathrm{C}$ for $114 \mathrm{~h}$ and quenched with methanol $(2.5 \mathrm{~mL})$. The mixture was passed through a short plug of silica $(\mathrm{EtOAc} / \mathrm{Hexane}=$ 1:2, then $10 \%$ triethylamine in EtOAc) to separate the alcohol and the acetate from the catalyst. The solution of alcohol and acetate was concentrated and the residue was purified by flash chromatography (Ether/Pentane $=1: 4)$, which provided $740 \mathrm{mg}$ of acetate $(S, R)-5 \mathrm{in} 47 \%$ yield. ${ }^{1} \mathrm{H}$ NMR $\left(400 \mathrm{MHz}, \mathrm{CDCl}_{3}\right) \delta 7.52$ (d, $1 \mathrm{H}, J=7.6 \mathrm{~Hz}$ ), 7.29 (ddd, $1 \mathrm{H}, J=6.8,6.8,1.6 \mathrm{~Hz}$ ), 7.23 (dd, 1H, $J=7.6,1.6 \mathrm{~Hz}$ ), $7.16(\mathrm{t}, 1 \mathrm{H}, J=6.8 \mathrm{~Hz}), 6.22(\mathrm{~s}, 1 \mathrm{H}), 5.98(\mathrm{dq}, 1 \mathrm{H}, J=16,1.6 \mathrm{~Hz})$, 5.39-5.31 (m, 1H), 4.97 (AB, 2H, $J=13.6 \mathrm{~Hz}), 3.69$ (s, 3H), 2.01 (s, 3H), 1.78 (dd, 3H, $J=6.0$, $1.6 \mathrm{~Hz}), 1.15$ (s, 3H), 0.97 (s, 9H), $0.15(\mathrm{~s}, 3 \mathrm{H}), 0.12(\mathrm{~s}, 3 \mathrm{H}) ;{ }^{13} \mathrm{C}\left(100 \mathrm{MHz}, \mathrm{CDCl}_{3}\right) \delta 175.0$, 169.5, 139.9, 133.1, 129.0, 128.3, 128.2, 127.6, 126.1, 125.8, 74.3, 62.1, 52.6, 52.3, 26.0, 20.9, 18.4, 18.3, 18.2, -5.3, -5.4; HRMS (APCI): $m / z$ calcd. for $\mathrm{C}_{23} \mathrm{H}_{37} \mathrm{O}_{5} \mathrm{Si}\left(\mathrm{M}^{+}\right)$421.2410, found 421.2399; FT-IR (KBr) 3034, 2953, 1741, $1231 \mathrm{~cm}^{-1} ;[\alpha]_{\mathrm{D}}^{25}=+17.1\left(\mathrm{c}=1.1, \mathrm{CHCl}_{3}\right)$.

$636 \mathrm{mg}$ of alcohol $(R, S)-\mathbf{3}$ was isolated in $45 \%$ yield and reverse phase HPLC analysis of the alcohol revealed $87 \%$ ee (Chiralpak AS-RH); $[\alpha]^{25}=-74.3\left(\mathrm{c}=1.2, \mathrm{CHCl}_{3}\right)$. 


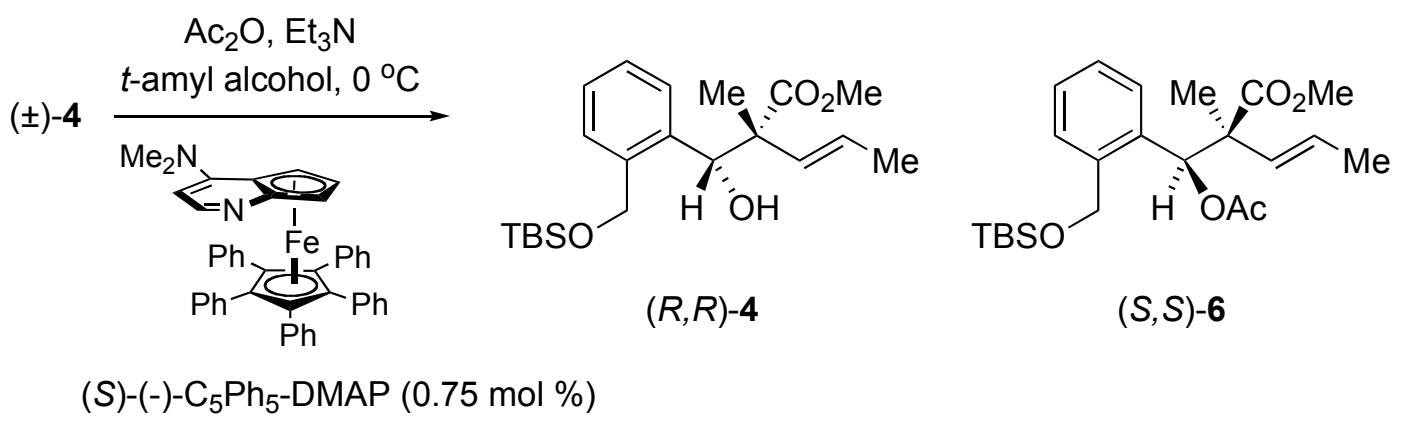

Kinetic resolution of 4 . The kinetic resolution procedure was followed using the racemic homoallylic alcohol $( \pm)-4(1.01 \mathrm{~g}, 2.67 \mathrm{mmol})$, catalyst $(S)-(-)-\mathrm{C}_{5} \mathrm{Ph}_{5}$-DMAP $(18 \mathrm{mg}, 0.03$ $\mathrm{mmol})$, triethylamine $(0.27 \mathrm{~mL}, 2.00 \mathrm{mmol})$, tert-amyl alcohol $(5.5 \mathrm{~mL})$ and acetic anhydride $(0.19 \mathrm{~mL}, 2.00 \mathrm{mmol})$. Reaction time: $119 \mathrm{~h}$. The crude mixture was purified by flash chromatography (Ether/Pentane $=1: 3$ ), which provided $499 \mathrm{mg}$ of acetate $(S, S)-6$ in $44 \%$ yield. ${ }^{1} \mathrm{H}$ NMR $\left(400 \mathrm{MHz}, \mathrm{CDCl}_{3}\right) \delta 7.57(\mathrm{~d}, 1 \mathrm{H}, J=8.0 \mathrm{~Hz}), 7.33-7.27(\mathrm{~m}, 2 \mathrm{H}), 7.22-7.19(\mathrm{~m}, 2 \mathrm{H})$, $6.24(\mathrm{~s}, 1 \mathrm{H}), 5.36-5.30(\mathrm{~m}, 2 \mathrm{H}), 4.90(\mathrm{AB}, 2 \mathrm{H}, J=13.6 \mathrm{~Hz}), 3.74(\mathrm{~s}, 3 \mathrm{H}), 2.00(\mathrm{~s}, 3 \mathrm{H}), 1.59$ (d, $3 \mathrm{H}, J=4.8 \mathrm{~Hz}), 1.35$ (s, 3H), 0.97 (s, 9H), $0.14(\mathrm{~s}, 3 \mathrm{H}), 0.11(\mathrm{~s}, 3 \mathrm{H}) ;{ }^{13} \mathrm{C}\left(100 \mathrm{MHz}, \mathrm{CDCl}_{3}\right) \delta$ 174.3, 169.2, 139.9, 132.7, 130.0, 128.0, 127.3, 127.2, 125.9, 125.6, 73.4, 61.8, 53.7, 52.2, 26.0, 20.9, 18.4, 18.2, 16.1, -5.3, -5.5; HRMS (APCI): $m / z$ calcd. for $\mathrm{C}_{23} \mathrm{H}_{37} \mathrm{O}_{5} \mathrm{Si}\left(\mathrm{M}^{+}\right)$421.2410, found 421.2397; FT-IR (KBr) 3034, 2995, 1745, $1231 \mathrm{~cm}^{-1} ;[\alpha]^{25}=-50.0\left(\mathrm{c}=0.23, \mathrm{CHCl}_{3}\right)$.

$461 \mathrm{mg}$ of alcohol $(R, R)-4$ was isolated in $46 \%$ yield and reverse phase HPLC analysis of the alcohol revealed $89 \%$ ee $\left(\right.$ Chiralpak AS-RH); $[\alpha]^{25}=+16.0\left(\mathrm{c}=0.3, \mathrm{CHCl}_{3}\right)$. 


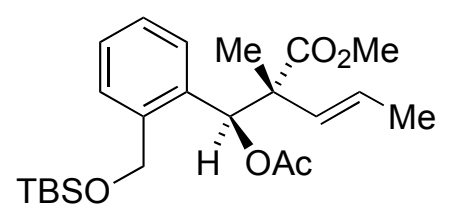

$(S, R)-5$

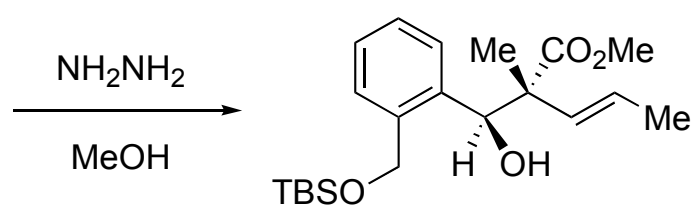

$(S, R)-3$

Basic methanolysis of 5. Acetate $(S, R)-5(665 \mathrm{mg}, 1.58 \mathrm{mmol})$ was dissolved in methanol (7 $\mathrm{mL}$ ) under argon at $0{ }^{\circ} \mathrm{C}$, and anhydrous hydrazine $(0.99 \mathrm{~mL}, 31.62 \mathrm{mmol})$ was added. The reaction was maintained at $0{ }^{\circ} \mathrm{C}$ for additional $3 \mathrm{~h}$ before quenching with saturated $\mathrm{CuSO}_{4(\mathrm{aq})}(5$ $\mathrm{mL}$ ). The precipitate was filtered off and the extractions were performed with ether. The combined organic layers were dried over $\mathrm{MgSO}_{4}$ and concentrated to afford a yellowish oil. The crude product was purified by flash chromatography on silica gel (Ether/Pentane $=1: 4)$ to yield alcohol $(S, R)-3$ (438 mg, 73\%); reverse phase HPLC analysis of the alcohol revealed $85 \%$ ee $($ Chiralpak AS-RH $) ;[\alpha]^{25}=+72.0\left(\mathrm{c}=2.8, \mathrm{CHCl}_{3}\right)$.

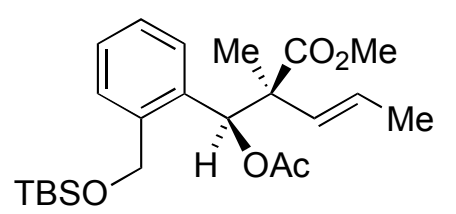

$(S, S)-6$

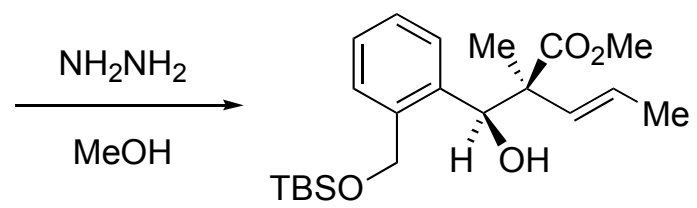

$(S, S)-4$

Basic methanolysis of 6. Acetate $(S, S)-6$ ( $463 \mathrm{mg}, 1.10 \mathrm{mmol}$ ) was dissolved in methanol (5 $\mathrm{mL})$ under argon at $0{ }^{\circ} \mathrm{C}$, and anhydrous hydrazine $(0.69 \mathrm{~mL}, 22.00 \mathrm{~mL})$ was added. The reaction was maintained at $0{ }^{\circ} \mathrm{C}$ for additional $3 \mathrm{~h}$ before quenching with saturated $\mathrm{CuSO}_{4}(\mathrm{aq})(4$ $\mathrm{mL}$ ). The precipitate was filtered off and the extractions were performed with ether. The combined organic layers were dried over $\mathrm{MgSO}_{4}$ and concentrated to afford a yellowish oil. The crude product was purified by flash chromatography on silica gel $($ Ether/Pentane $=1: 3)$ to yield alcohol $(S, S)$-4 (314 mg, 75\%); reverse phase HPLC analysis of the alcohol revealed $90 \%$ ee (Chiralpak AS-RH); $[\alpha]^{25}=-17.7\left(\mathrm{c}=2.8, \mathrm{CHCl}_{3}\right)$. 


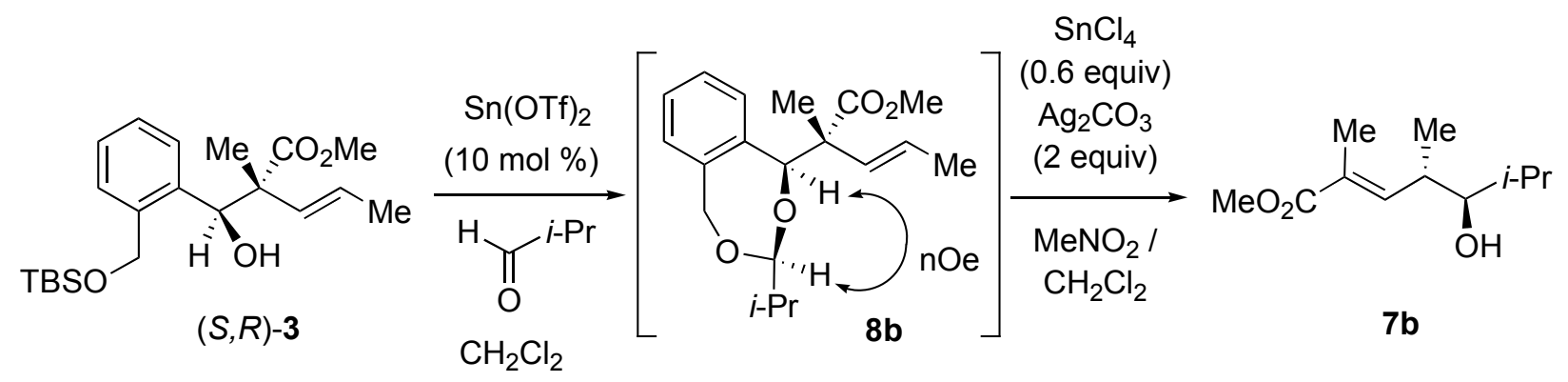

$(4 S, 5 R)$-5-Hydroxy-2,4,6-trimethylhept-2-enoic acid methyl ester (7b). General procedure

for bispropionate transfer (simple aldehydes, Scheme 2): $\mathrm{Sn}(\mathrm{OTf})_{2}(13 \mathrm{mg}, 0.032 \mathrm{mmol})$ was added to a solution of alcohol $(S, R)-3(120 \mathrm{mg}, 0.32 \mathrm{mmol})$ and freshly distilled isobutyraldehyde $(27 \mathrm{mg}, 0.38 \mathrm{mmol})$ in $\mathrm{CH}_{2} \mathrm{Cl}_{2}(3.2 \mathrm{~mL})$ under argon at room temperature. The resulting solution was stirred at room temperature for $1 \mathrm{~h}$ and passed through a short plug of silica $($ EtOAc/Hexane $=1: 4)$. The solvents were removed by rotary evaporation and the crude cyclic acetal $\mathbf{8 b ^ { 3 }}$ was used for the next step without further purification. The crude acetal $\mathbf{8 b}$ was dissolved in $\mathrm{MeNO}_{2}(1.6 \mathrm{~mL})$ and $\mathrm{CH}_{2} \mathrm{Cl}_{2}(1.6 \mathrm{~mL})$ under argon, and $\mathrm{Ag}_{2} \mathrm{CO}_{3}(174 \mathrm{mg}, 0.64$ mmol) and $\mathrm{SnCl}_{4}\left(0.19 \mathrm{~mL}, 1 \mathrm{M}\right.$ in $\left.\mathrm{CH}_{2} \mathrm{Cl}_{2}, 0.19 \mathrm{mmol}\right)$ were added at room temperature. The resulting solution was stirred at room temperature for $75 \mathrm{~min}$ before quenching with saturated aqueous $\mathrm{NaHCO}_{3}(3 \mathrm{~mL})$. The salts were filtered off with Celite and the extractions were performed with $\mathrm{CH}_{2} \mathrm{Cl}_{2}$. The combined organic layers were dried $\left(\mathrm{MgSO}_{4}\right)$, filtered, and concentrated. The residue was purified by flash chromatography on silica gel (Ether/Pentane $=$ 1:1) to yield alcohol $7 \mathbf{b}(47 \mathrm{mg}, 73 \%)$. ${ }^{1} \mathrm{H}$ NMR (400 MHz, $\left.\mathrm{CDCl}_{3}\right) \delta 6.76(\mathrm{dq}, 1 \mathrm{H}, J=10.4,1.6$ $\mathrm{Hz}), 3.72(\mathrm{~s}, 3 \mathrm{H}), 3.22$ (t, 1H, $J=5.6 \mathrm{~Hz}), 2.72-2.67(\mathrm{~m}, 1 \mathrm{H}), 1.87$ (d, 3H, $J=1.2 \mathrm{~Hz}), 1.73-1.65$ (m, 1H), 1.59 (br s, 1H), $1.01(\mathrm{~d}, 3 \mathrm{H}, J=6.8 \mathrm{~Hz}), 0.93$ (d, 3H, $J=6.8 \mathrm{~Hz}), 0.90$ (d, 3H, $J=6.4$ $\mathrm{Hz}) ;{ }^{13} \mathrm{C}\left(100 \mathrm{MHz} \mathrm{CDCl}_{3}\right) \delta 168.6,143.8,127.9,80.1,51.7,36.5,30.9,19.6,16.8,16.7,12.7$; HRMS (APCI): $m / z$ calcd. for $\mathrm{C}_{11} \mathrm{H}_{21} \mathrm{O}_{3}\left(\mathrm{M}^{+}\right)$201.1491, found 201.1485; FT-IR (KBr) 3497, 2960, 2874, 1712, 1648, $1285 \mathrm{~cm}^{-1} ;[\alpha]^{25}=-12.8\left(\mathrm{c}=0.7, \mathrm{CHCl}_{3}\right)$; Mosher ester analysis of the alcohol revealed 11:1 er ( $83 \%$ ee).

(3) Acetal $\mathbf{8 b}$ is a single diastereomer and the relative stereochemistry was confirmed by nOe (nuclear Overhauser effect) experiment. ${ }^{1} \mathrm{H}$ NMR $\left(400 \mathrm{MHz} \mathrm{CDCl}_{3}\right) \delta$ 7.20-7.17 (m, 3H), 7.14-7.12 (m, 1H), $5.93(\mathrm{dq}, 1 \mathrm{H}, J=16,1.6$ $\mathrm{Hz}), 5.47-5.39(\mathrm{~m}, 1 \mathrm{H}), 5.24(\mathrm{~s}, 1 \mathrm{H}), 4.83(\mathrm{AB}, 2 \mathrm{H}, J=13.0 \mathrm{~Hz}), 4.54(\mathrm{~d}, 1 \mathrm{H}, J=5.6 \mathrm{~Hz}), 3.71(\mathrm{~s}, 3 \mathrm{H}), 1.87-1.79$ $(\mathrm{m}, 1 \mathrm{H}), 1.68(\mathrm{dd}, 3 \mathrm{H}, J=6.4,1.6 \mathrm{~Hz}), 1.42(\mathrm{~s}, 3 \mathrm{H}), 0.91(\mathrm{~d}, 6 \mathrm{H}, J=7.2 \mathrm{~Hz}) ;{ }^{13} \mathrm{C}\left(100 \mathrm{MHz}, \mathrm{CDCl}_{3}\right) \delta 175.4,138.8$, 137.2, 131.7, 129.6, 127.6, 127.3, 126.6, 125.9, 107.7, 85.6, 69.4, 53.2, 52.2, 33.1, 18.2, 17.8, 17.6, 17.0. HRMS (APCI): $m / z$ calcd. for $\mathrm{C}_{19} \mathrm{H}_{27} \mathrm{O}_{4}\left(\mathrm{M}^{+}\right) 319.1909$, found 319.1904; FT-IR (KBr) 2956, 1732, $1452,1243 \mathrm{~cm}^{-1}$. 


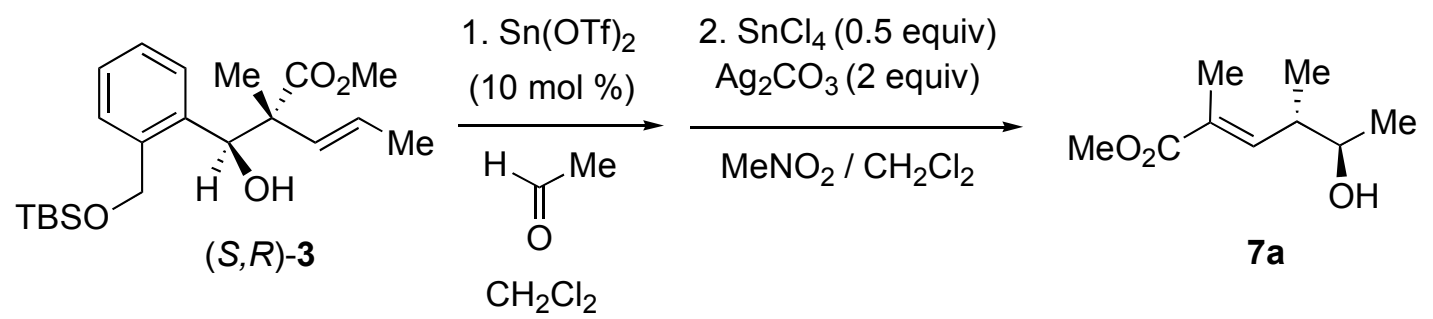

(4S, 5R)-5-Hydroxy-2,4-dimethylhex-2-enoic acid methyl ester (7a). The general procedure was followed using $\mathrm{Sn}(\mathrm{OTf})_{2}(13 \mathrm{mg}, 0.03 \mathrm{mmol})$, alcohol $(S, R)-3$ (100 mg, $\left.0.26 \mathrm{mmol}\right)$, acetaldehyde (14 mg, $0.32 \mathrm{mmol}), \mathrm{Ag}_{2} \mathrm{CO}_{3}(143 \mathrm{mg}, 0.52 \mathrm{mmol})$ and $\mathrm{SnCl}_{4}(0.13 \mathrm{~mL}, 1 \mathrm{M}$ in $\left.\mathrm{CH}_{2} \mathrm{Cl}_{2}, 0.13 \mathrm{mmol}\right)$. The residue was purified by flash chromatography on silica gel $($ Ether/Pentane $=1: 1)$ to yield homoallylic alcohol $7 \mathbf{a}(30 \mathrm{mg}, 67 \%) .{ }^{1} \mathrm{H} \mathrm{NMR}\left(400 \mathrm{MHz}, \mathrm{CDCl}_{3}\right)$ $\delta 6.67(\mathrm{dq}, 1 \mathrm{H}, J=10.4,1.4 \mathrm{~Hz}), 3.75(\mathrm{~s}, 3 \mathrm{H}), 3.73-3.67(\mathrm{~m}, 1 \mathrm{H}), 2.57-2.48(\mathrm{~m}, 1 \mathrm{H}), 1.88(\mathrm{~d}, 3 \mathrm{H}$, $J=1.4 \mathrm{~Hz}), 1.20(\mathrm{~d}, 3 \mathrm{H}, J=6.4 \mathrm{~Hz}), 1.03(\mathrm{~d}, 3 \mathrm{H}, J=6.4 \mathrm{~Hz}) ;{ }^{13} \mathrm{C}\left(100 \mathrm{MHz}, \mathrm{CDCl}_{3}\right) \delta 168.5$, 143.8, 128.8, 71.3, 51.8, 40.9, 20.7, 16.1, 12.8; HRMS (APCI): $m / z$ calcd. for $\mathrm{C}_{9} \mathrm{H}_{17} \mathrm{O}_{3}\left(\mathrm{M}^{+}\right)$ 173.1178, found 173.1173; FT-IR (KBr) 3459, 2972, 2876, 1710, 1648, 1436, $1243 \mathrm{~cm}^{-1}$; $[\alpha]^{25}{ }_{\mathrm{D}}$ $=-28\left(\mathrm{c}=0.13, \mathrm{CHCl}_{3}\right) ;$ Mosher ester analysis of the alcohol revealed 11:1 er $(83 \% \mathrm{ee})$.

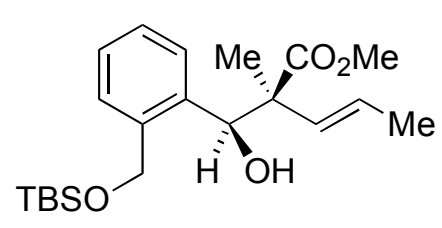

$(S, S)-4$

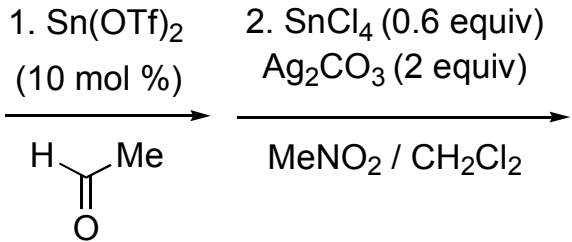

$\mathrm{CH}_{2} \mathrm{Cl}_{2}$

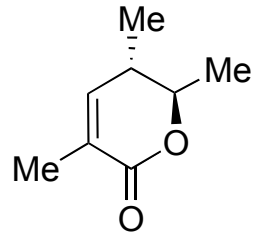

$10 a$

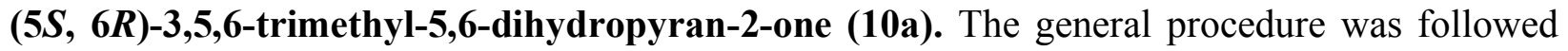
using $\mathrm{Sn}(\mathrm{OTf})_{2}(8 \mathrm{mg}, 0.02 \mathrm{mmol})$, alcohol $(S, S)-4(55 \mathrm{mg}, 0.13 \mathrm{mmol})$, acetaldehyde $(8 \mathrm{mg}$, $0.18 \mathrm{mmol}), \mathrm{Ag}_{2} \mathrm{CO}_{3}(72 \mathrm{mg}, 0.26 \mathrm{mmol})$ and $\mathrm{SnCl}_{4}\left(0.07 \mathrm{~mL}, 1 \mathrm{M}\right.$ in $\left.\mathrm{CH}_{2} \mathrm{Cl}_{2}, 0.07 \mathrm{mmol}\right)$. The residue was purified by flash chromatography on silica gel (Ether/Pentane $=1: 2)$ to yield $\delta$ lactone 10a (17 mg, 94\%). ${ }^{1} \mathrm{H}$ NMR (400 MHz, $\left.\mathrm{CDCl}_{3}\right) \delta$ 6.33-6.32 (m, 1H), 4.16-4.09 (m, 1H), 2.43-2.36 (m, 1H), 1.91-1.90 (m, 3H), $1.40(\mathrm{~d}, 3 \mathrm{H}, J=6.0 \mathrm{~Hz}), 1.09(\mathrm{~d}, 3 \mathrm{H}, J=7.2 \mathrm{~Hz}) ;{ }^{13} \mathrm{C}$ $\left(150 \mathrm{MHz}, \mathrm{CDCl}_{3}\right) \delta 166.0,145.6,127.3,80.1,35.6,19.1,16.8,16.4 ; \mathrm{HRMS}$ (APCI): $\mathrm{m} / z$ calcd. for $\mathrm{C}_{8} \mathrm{H}_{13} \mathrm{O}_{2}\left(\mathrm{M}^{+}\right)$141.0916, found 141.0909; FT-IR (KBr) 2980, 2883, 1718, $1451 \mathrm{~cm}^{-1}$; $[\alpha]^{25}=$ $-45\left(\mathrm{c}=0.1, \mathrm{CHCl}_{3}\right)$; normal phase HPLC analysis of the lactone revealed $88 \%$ ee (Chiralpak AS). 


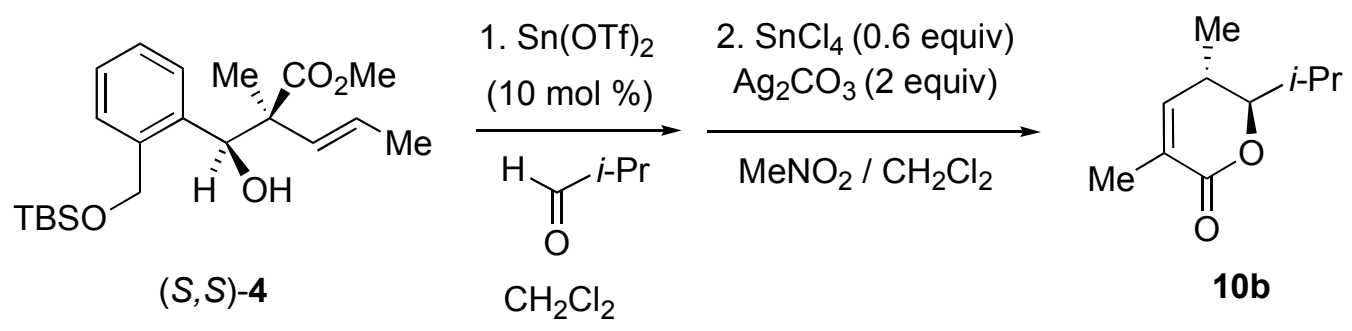

(5S, 6R)-6-Isopropyl-3,5-dimethyl-5,6-dihydropyran-2-one (10b). The general procedure was followed using $\mathrm{Sn}(\mathrm{OTf})_{2}(8 \mathrm{mg}, 0.02 \mathrm{mmol})$, alcohol $(S, S)-4(76 \mathrm{mg}, 0.20 \mathrm{mmol})$, freshly distilled isobutyraldehyde (15 mg, $0.20 \mathrm{mmol}), \mathrm{Ag}_{2} \mathrm{CO}_{3}(109 \mathrm{mg}, 0.40 \mathrm{mmol})$ and $\mathrm{SnCl}_{4}(0.12$ $\mathrm{mL}, 1 \mathrm{M}$ in $\mathrm{CH}_{2} \mathrm{Cl}_{2}, 0.12 \mathrm{mmol}$ ). The residue was purified by flash chromatography on silica gel $($ Ether/Pentane $=1: 5)$ to yield $\delta$-lactone 10b $(31 \mathrm{mg}, 92 \%) .{ }^{1} \mathrm{H}$ NMR $\left(400 \mathrm{MHz}, \mathrm{CDCl}_{3}\right) \delta 6.32-$ $6.30(\mathrm{~m}, 1 \mathrm{H}), 3.85(\mathrm{dd}, 1 \mathrm{H}, J=10.0,2.8 \mathrm{~Hz}), 2.58-2.52(\mathrm{~m}, 1 \mathrm{H}), 1.96-1.90(\mathrm{~m}, 1 \mathrm{H}), 1.87(\mathrm{dd}$, $3 \mathrm{H}, J=2.0,1.2 \mathrm{~Hz}), 1.06$ (d, 3H, $J=4.0 \mathrm{~Hz}), 1.04$ (d, 3H, $J=4.0 \mathrm{~Hz}), 0.94$ (d, $3 \mathrm{H}, J=6.8 \mathrm{~Hz})$; ${ }^{13} \mathrm{C}\left(100 \mathrm{MHz}, \mathrm{CDCl}_{3}\right) \delta 166.1,146.1,127.1,87.8,31.1,28.9,19.6,16.8,16.4,15.3$; HRMS (APCI): $m / z$ calcd. for $\mathrm{C}_{10} \mathrm{H}_{17} \mathrm{O}_{2}\left(\mathrm{M}^{+}\right)$169.1229, found 169.1222; FT-IR (KBr) 2968, 2880, 1718 , $1459 \mathrm{~cm}^{-1} ;[\alpha]^{25}=+23.5\left(\mathrm{c}=1.4, \mathrm{CHCl}_{3}\right)$; normal phase HPLC analysis of the lactone revealed $89 \%$ ee (Chiralpak AS). 


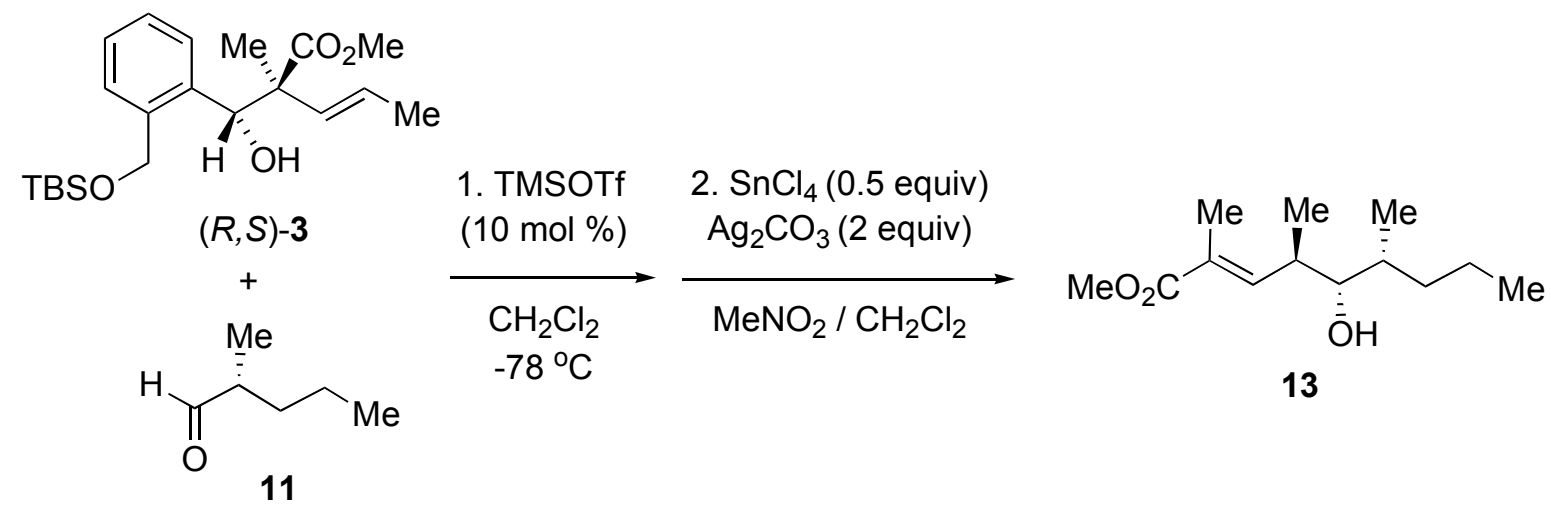

$(4 R, 5 S, 6 R)$-5-Hydroxy-2,4,6-trimethylnon-2-enoic acid methyl ester (13). General procedure A for bispropionate transfer to alpha-chiral aldehydes (Table 1): Alcohol $(R, S)$-3 $(50 \mathrm{mg}, 0.13 \mathrm{mmol})$ and $(2 R)-2$-methylpentanal $11(16 \mathrm{mg}, 0.16 \mathrm{mmol})$ were dissolved in $\mathrm{CH}_{2} \mathrm{Cl}_{2}\left(1.3 \mathrm{~mL}\right.$ ), cooled to $-78{ }^{\circ} \mathrm{C}$, and TMSOTf ( $\left.3 \mathrm{mg}, 0.013 \mathrm{mmol}\right)$ was added dropwise. The resulting solution was stirred at $-78{ }^{\circ} \mathrm{C}$ for $4 \mathrm{~h}$ and quenched with pyridine $(10 \mu \mathrm{L})$. The reaction mixture was passed through a short plug of silica $($ EtOAc/Hexane $=1: 4)$. The solvents were removed by rotary evaporation and the residue was used for the next transformation without further purification. The crude acetal intermediate was dissolved in $\mathrm{MeNO}_{2}(0.7 \mathrm{~mL})$ and $\mathrm{CH}_{2} \mathrm{Cl}_{2}$ (0.7 mL) under argon, and $\mathrm{Ag}_{2} \mathrm{CO}_{3}(72 \mathrm{mg}, 0.26 \mathrm{mmol})$ and $\mathrm{SnCl}_{4}\left(0.065 \mathrm{~mL}, 1 \mathrm{M}\right.$ in $\mathrm{CH}_{2} \mathrm{Cl}_{2}$, $0.065 \mathrm{mmol}$ ) were added at room temperature. The resulting solution was stirred at room temperature for $75 \mathrm{~min}$ before quenched with saturated aqueous $\mathrm{NaHCO}_{3}(3 \mathrm{~mL})$. The salts were filtered off with celite and the extractions were performed with $\mathrm{CH}_{2} \mathrm{Cl}_{2}$. The combined organic layers were dried $\left(\mathrm{MgSO}_{4}\right)$, filtered, and concentrated. The residue was purified by flash chromatography on silica gel (Ether/Pentane $=1: 3)$ to yield homoallylic alcohol $13(22 \mathrm{mg}$, 75\%). ${ }^{1} \mathrm{H}$ NMR analysis of the alcohol revealed $12: 1 \mathrm{dr} .{ }^{1} \mathrm{H}$ NMR $\left(400 \mathrm{MHz}, \mathrm{CDCl}_{3}\right) \delta 6.71(\mathrm{dq}$, $1 \mathrm{H}, J=10.0,1.2 \mathrm{~Hz}$ ), 3.73 (s, 3H), 3.35 (dd, $1 \mathrm{H}, J=7.4,4.0 \mathrm{~Hz}), 2.73-2.67$ (m, $1 \mathrm{H}), 1.88$ (d, 3H, $J=1.2 \mathrm{~Hz}), 1.64-1.58(\mathrm{~m}, 1 \mathrm{H}), 1.46(\mathrm{br} \mathrm{s}, 1 \mathrm{H}), 1.39-1.18(\mathrm{~m}, 4 \mathrm{H}), 0.97$ (d, 3H, $J=6.8 \mathrm{~Hz})$, $0.92-0.87(\mathrm{~m}, 6 \mathrm{H}) ;{ }^{13} \mathrm{C}\left(100 \mathrm{MHz}, \mathrm{CDCl}_{3}\right) \delta 168.6,144.7,128.2,78.1,51.8,36.8,36.2,34.7$, 20.2, 16.4, 14.3, 12.9, 12.8; HRMS (APCI): $m / z$ calcd. for $\mathrm{C}_{13} \mathrm{H}_{25} \mathrm{O}_{3}\left(\mathrm{M}^{+}\right)$229.1804, found $211.1693\left(\mathrm{M}^{+}-\mathrm{H}_{2} \mathrm{O}\right)$; FT-IR (KBr) 3494, 2963, 2879, 1710, 1645, 1438, $1240 \mathrm{~cm}^{-1}$. 


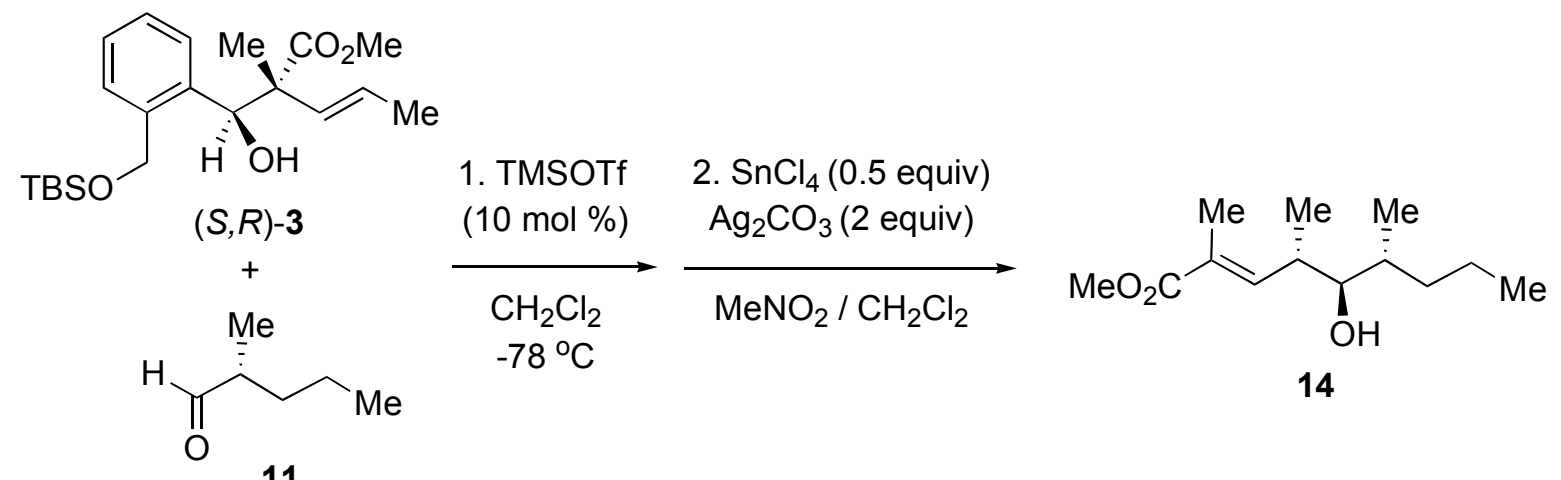

$(4 S, 5 R, 6 R)$-5-Hydroxy-2,4,6-trimethylnon-2-enoic acid methyl ester (14). The general procedure A was followed using TMSOTf (4 mg, $0.018 \mathrm{mmol}$ ), alcohol $(S, R)-3$ (68 mg, 0.18 mmol), (2R)-2-methylpentanal 11 (22 mg, $0.22 \mathrm{mmol}), \mathrm{Ag}_{2} \mathrm{CO}_{3}(99 \mathrm{mg}, 0.36 \mathrm{mmol})$ and $\mathrm{SnCl}_{4}$ (0.09 $\mathrm{mL}, 1 \mathrm{M}$ in $\mathrm{CH}_{2} \mathrm{Cl}_{2}, 0.09 \mathrm{mmol}$ ). The residue was purified by flash chromatography on silica gel $($ Ether/Pentane $=1: 3)$ to yield homoallylic alcohol $14(32 \mathrm{mg}, 78 \%) .{ }^{1} \mathrm{H}$ NMR analysis of the alcohol revealed 10:1 dr. ${ }^{1} \mathrm{H}$ NMR $\left(400 \mathrm{MHz}, \mathrm{CDCl}_{3}\right) \delta 6.77(\mathrm{dq}, 1 \mathrm{H}, J=10.0,1.6 \mathrm{~Hz})$, $3.74(\mathrm{~s}, 3 \mathrm{H}), 3.29$ (dd, $1 \mathrm{H}, J=6.0,5.6 \mathrm{~Hz}), 2.77-2.70(\mathrm{~m}, 1 \mathrm{H}), 1.88(\mathrm{~d}, 3 \mathrm{H}, J=1.6 \mathrm{~Hz}), 1.56-$ 1.40 (m, 3H), 1.27-1.10 (m, 2H), 1.03 (d, 3H, $J=7.2 \mathrm{~Hz}), 0.91$ (t, 3H, $J=7.2 \mathrm{~Hz}), 0.88$ (d, 3H, $J$ $=6.8 \mathrm{~Hz}) ;{ }^{13} \mathrm{C}\left(100 \mathrm{MHz}, \mathrm{CDCl}_{3}\right) \delta 168.9,143.9,128.1,79.9,52.0,36.5,36.1,33.5,20.3,17.2$, 16.5, 14.7, 12.9; HRMS (APCI): $m / z$ calcd. for $\mathrm{C}_{13} \mathrm{H}_{25} \mathrm{O}_{3}\left(\mathrm{M}^{+}\right)$229.1804, found $211.1692\left(\mathrm{M}^{+}-\right.$ $\mathrm{H}_{2} \mathrm{O}$ ); FT-IR (KBr) 3497, 2961, 2876, 1710, 1648, 1436, $1239 \mathrm{~cm}^{-1}$.

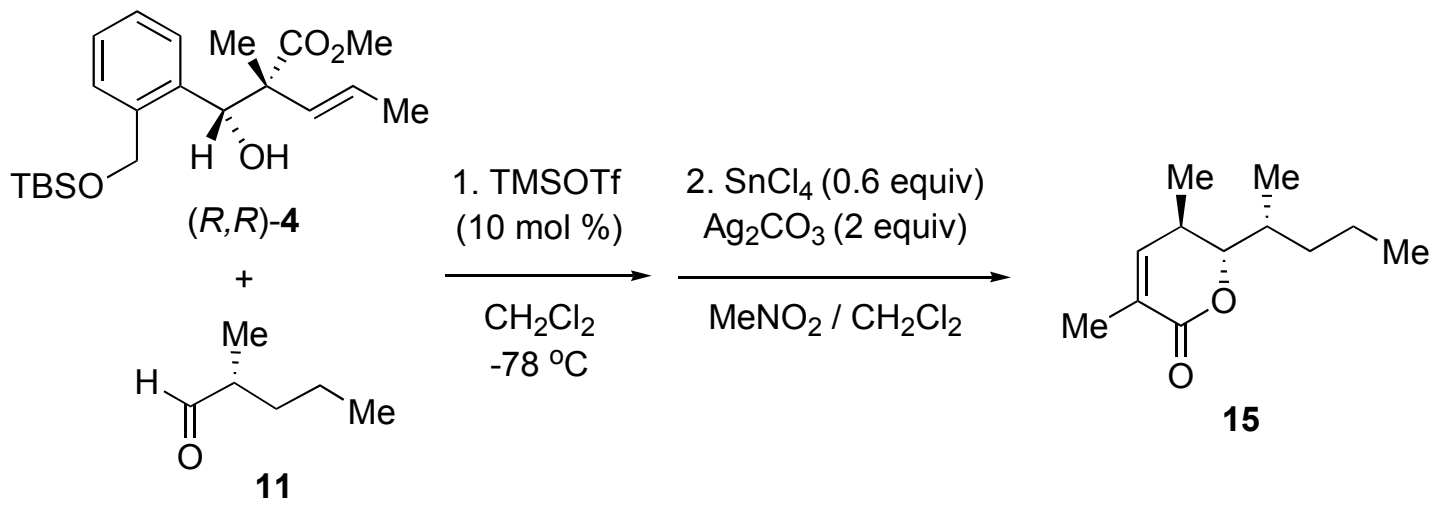

(5R, 6S, 1'R)-3,5-Dimethyl-6-(1'-methylbutyl)-5,6-dihydropyran-2-one (15). The general procedure A was followed using TMSOTf $(7 \mathrm{mg}, 0.03 \mathrm{mmol})$, alcohol $(R, R)-4(107 \mathrm{mg}, 0.28$ mmol), (2R)-2-methylpentanal 11 (38 mg, $0.38 \mathrm{mmol}), \mathrm{Ag}_{2} \mathrm{CO}_{3}(154 \mathrm{mg}, 0.56 \mathrm{mmol})$ and $\mathrm{SnCl}_{4}$ (0.17 $\mathrm{mL}, 1 \mathrm{M}$ in $\mathrm{CH}_{2} \mathrm{Cl}_{2}, 0.17 \mathrm{mmol}$ ). The residue was purified by flash chromatography on 
silica gel $($ Ether/Pentane $=1: 5)$ to yield $\delta$-lactone $15(50 \mathrm{mg}, 89 \%) .{ }^{1} \mathrm{H}$ NMR analysis of the lactone revealed 14:1 dr; ${ }^{1} \mathrm{H}$ NMR $\left(400 \mathrm{MHz}, \mathrm{CDCl}_{3}\right) \delta$ 6.34-6.33 (m, 1H), $3.97(\mathrm{dd}, 1 \mathrm{H}, J=$ 10.8, $2.4 \mathrm{~Hz}), 2.66-2.58(\mathrm{~m}, 1 \mathrm{H}), 1.91-1.90(\mathrm{~m}, 3 \mathrm{H}), 1.76-1.70(\mathrm{~m}, 1 \mathrm{H}), 1.52-1.29(\mathrm{~m}, 4 \mathrm{H}), 1.05$ $(\mathrm{d}, 3 \mathrm{H}, J=7.2 \mathrm{~Hz}), 0.94(\mathrm{~d}, 3 \mathrm{H}, J=6.8 \mathrm{~Hz}), 0.90(\mathrm{t}, 3 \mathrm{H}, J=7.6 \mathrm{~Hz}) ;{ }^{13} \mathrm{C}\left(100 \mathrm{MHz}, \mathrm{CDCl}_{3}\right) \delta$ 166.3, 146.4, 127.1, 86.1, 35.6, 33.4, 31.0, 20.4, 16.8, 16.2, 14.1, 13.1; FT-IR (KBr) 2961, 1718, $1459,1382 \mathrm{~cm}^{-1}$; HRMS (APCI): $\mathrm{m} / z$ calcd. for $\mathrm{C}_{12} \mathrm{H}_{21} \mathrm{O}_{2}\left(\mathrm{M}^{+}\right)$197.1542, found 197.1534; $[\alpha]^{25}$ $=-28.9\left(\mathrm{c}=0.40, \mathrm{CHCl}_{3}\right)$.

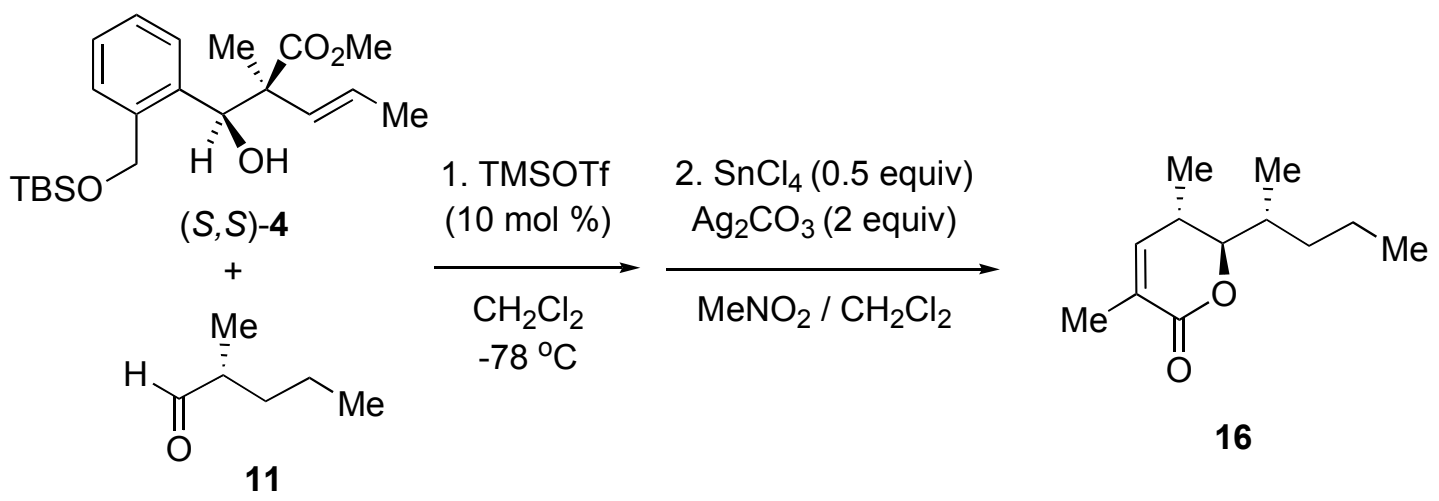

(5S, 6R, 1'R)-3,5-Dimethyl-6-(1'-methylbutyl)-5,6-dihydropyran-2-one (16). The general procedure A was followed using TMSOTf (3 mg, $0.013 \mathrm{mmol}$ ), alcohol $(S, S)-4$ (50 mg, 0.13 mmol), (2R)-2-methylpentanal 11 (16 mg, $0.16 \mathrm{mmol}), \mathrm{Ag}_{2} \mathrm{CO}_{3}(72 \mathrm{mg}, 0.26 \mathrm{mmol})$ and $\mathrm{SnCl}_{4}$ ( $0.065 \mathrm{~mL}, 1 \mathrm{M}$ in $\mathrm{CH}_{2} \mathrm{Cl}_{2}, 0.065 \mathrm{mmol}$ ). The residue was purified by flash chromatography on silica gel $($ Ether/Pentane $=1: 5)$ to yield yield $\delta$-lactone $16(22 \mathrm{mg}, 85 \%) .{ }^{1} \mathrm{H}$ NMR analysis of the lactone revealed 14:1 dr; ${ }^{1} \mathrm{H}$ NMR $\left(400 \mathrm{MHz}, \mathrm{CDCl}_{3}\right) \delta 6.35-6.33(\mathrm{~m}, 1 \mathrm{H}), 3.92(\mathrm{dd}, 1 \mathrm{H}, J=$ 9.6, 3.2 Hz), 2.69-2.60 (m, 1H), 1.90-1.89 (m, 3H), 1.82-1.77 (m, 1H), 1.52-1.18 (m, 4H), 1.07 $(\mathrm{d}, 3 \mathrm{H}, J=7.2 \mathrm{~Hz}), 1.05(\mathrm{~d}, 3 \mathrm{H}, J=6.8 \mathrm{~Hz}), 0.90(\mathrm{t}, 3 \mathrm{H}, J=7.2 \mathrm{~Hz}) ;{ }^{13} \mathrm{C}\left(100 \mathrm{MHz}, \mathrm{CDCl}_{3}\right) \delta$ 166.1, 146.1, 127.0, 88.2, 33.7, 32.0, 30.7, 20.4, 16.8, 16.7, 16.6, 14.2; FT-IR (KBr) 2964, 1718, 1459, $1135 \mathrm{~cm}^{-1}$; HRMS (APCI): $m / z$ calcd. for $\mathrm{C}_{12} \mathrm{H}_{21} \mathrm{O}_{2}\left(\mathrm{M}^{+}\right)$197.1542, found 197.1535. 


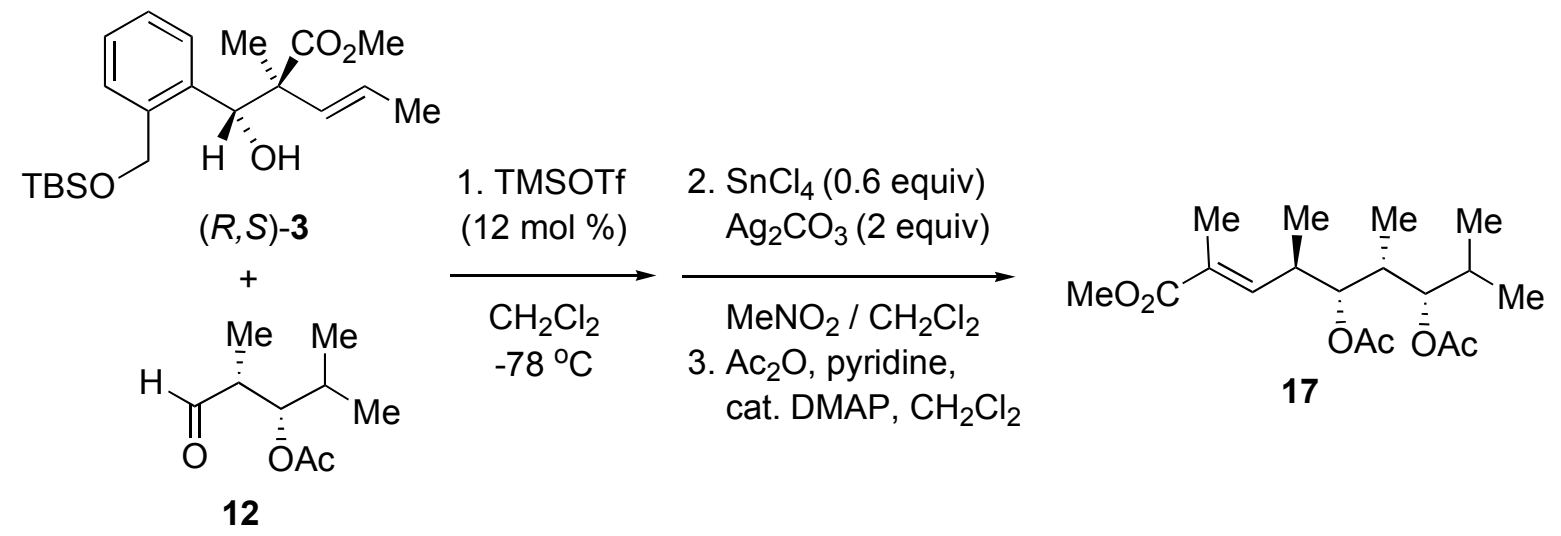

$(4 R, 5 R, 6 R, 7 S)$-5,7-Diacetoxy-2,4,6,8-tetramethylnon-2-enoic acid methyl ester (17). General procedure B for bispropionate transfer (Table 1): Alcohol $(R, S)-3$ (60 mg, 0.16 $\mathrm{mmol})$ and $(2 R, 3 S)$-3-acetoxy-2,4-dimethylpentanal $12(30 \mathrm{mg}, 0.17 \mathrm{mmol})$ were dissolved in $\mathrm{CH}_{2} \mathrm{Cl}_{2}(1.6 \mathrm{~mL})$, cooled to $-78^{\circ} \mathrm{C}$, and TMSOTf $(5 \mathrm{mg}, 0.02 \mathrm{mmol})$ was added dropwise. The resulting solution was stirred at $-78{ }^{\circ} \mathrm{C}$ for $4 \mathrm{~h}$ and quenched with pyridine $(10 \mu \mathrm{L})$. The reaction mixture was passed through a short plug of silica $($ EtOAc/Hexane $=1: 4)$. The solvents were removed by rotary evaporation and the residue was used for next transformation without further purification. The crude acetal intermediate was dissolved in $\mathrm{MeNO}_{2}(0.8 \mathrm{~mL})$ and $\mathrm{CH}_{2} \mathrm{Cl}_{2}(0.8$ $\mathrm{mL})$ under argon atmosphere, and $\mathrm{Ag}_{2} \mathrm{CO}_{3}(88 \mathrm{mg}, 0.32 \mathrm{mmol})$ and $\mathrm{SnCl}_{4}(0.1 \mathrm{~mL}, 1 \mathrm{M}$ in $\mathrm{CH}_{2} \mathrm{Cl}_{2}, 0.1 \mathrm{mmol}$ ) were added at room temperature. The resulting solution was stirred at room temperature for $1.5 \mathrm{~h}$ before quenching with saturated aqueous $\mathrm{NaHCO}_{3}(3 \mathrm{~mL})$. The salts were filtered off with celite and the extractions were performed with $\mathrm{CH}_{2} \mathrm{Cl}_{2}$. The combined organic layers were dried $\left(\mathrm{MgSO}_{4}\right)$, filtered, and concentrated. The residue was purified by flash chromatography on silica gel $(\mathrm{EtOAc} / \mathrm{Hexane}=1: 2)$ to yield homoallylic alcohol $(35 \mathrm{mg}, 73 \%)$. ${ }^{1} \mathrm{H}$ NMR analysis of the alcohol revealed a 3:2 mixture indicating partial migration of the acetate protective group. The alcohol mixture $(26 \mathrm{mg}, 0.09 \mathrm{mmol})$ in a flask was charged with argon and added $\mathrm{CH}_{2} \mathrm{Cl}_{2}(1 \mathrm{~mL})$, pyridine $(29 \mu \mathrm{L}, 0.36 \mathrm{mmol})$, acetic anhydride $(25 \mu \mathrm{L})$ and DMAP $(1 \mathrm{mg}$, $0.0045 \mathrm{mmol}$ ) at $0{ }^{\circ} \mathrm{C}$. After the addition, the resulting solution was allowed to warm to room temperature and stirred for another $2 \mathrm{~h}$ before quenching with water. The extractions was performed with $\mathrm{CH}_{2} \mathrm{Cl}_{2}$ and the combined organic layers were dried $\left(\mathrm{MgSO}_{4}\right)$, filtered, and concentrated. The residue was purified by flash chromatography on silica gel (EtOAc/Hexane $=$ $1: 4)$ to yield diacetate 17 in $95 \%$ yield. ${ }^{1} \mathrm{H}$ NMR $\left(400 \mathrm{MHz}, \mathrm{CDCl}_{3}\right) \delta 6.69(\mathrm{dq}, 1 \mathrm{H}, J=10.8$, $1.6 \mathrm{~Hz}), 4.81$ (dd, $1 \mathrm{H}, J=8.0,4.4 \mathrm{~Hz}), 4.62$ (dd, $1 \mathrm{H}, J=8.0,3.6 \mathrm{~Hz}), 3.75(\mathrm{~s}, 3 \mathrm{H}), 3.11-3.05$ (m, 
1H), 2.10 (s, 3H), 2.08 (s, 3H), 1.93(d, 3H, $J=1.6 \mathrm{~Hz}), 1.94-1.97(\mathrm{~m}, 2 \mathrm{H}), 0.94$ (d, 3H, $J=6.8$ $\mathrm{Hz}), 0.87(\mathrm{~d}, 3 \mathrm{H}, J=6.8 \mathrm{~Hz}), 0.84(\mathrm{~d}, 3 \mathrm{H}, J=6.8 \mathrm{~Hz}), 0.77(\mathrm{~d}, 3 \mathrm{H}, J=6.8 \mathrm{~Hz}) ;{ }^{13} \mathrm{C}(100 \mathrm{MHz}$, $\left.\mathrm{CDCl}_{3}\right) \delta 171.1,170.7,168.5,141.9,128.4,77.9,51.9,36.8,34.7,29.6,20.9,20.8,19.0,18.4$, 16.7, 12.7, 9.5; HRMS (APCI): $m / z$ calcd. for $\mathrm{C}_{18} \mathrm{H}_{31} \mathrm{O}_{6}\left(\mathrm{M}^{+}\right)$343.2121, found $283.1905\left(\mathrm{M}^{+}-\right.$ HOAc); FT-IR (KBr) 2972, 1738, $1237 \mathrm{~cm}^{-1} ;[\alpha]^{25}=-15.9\left(\mathrm{c}=1.04, \mathrm{CHCl}_{3}\right)$.

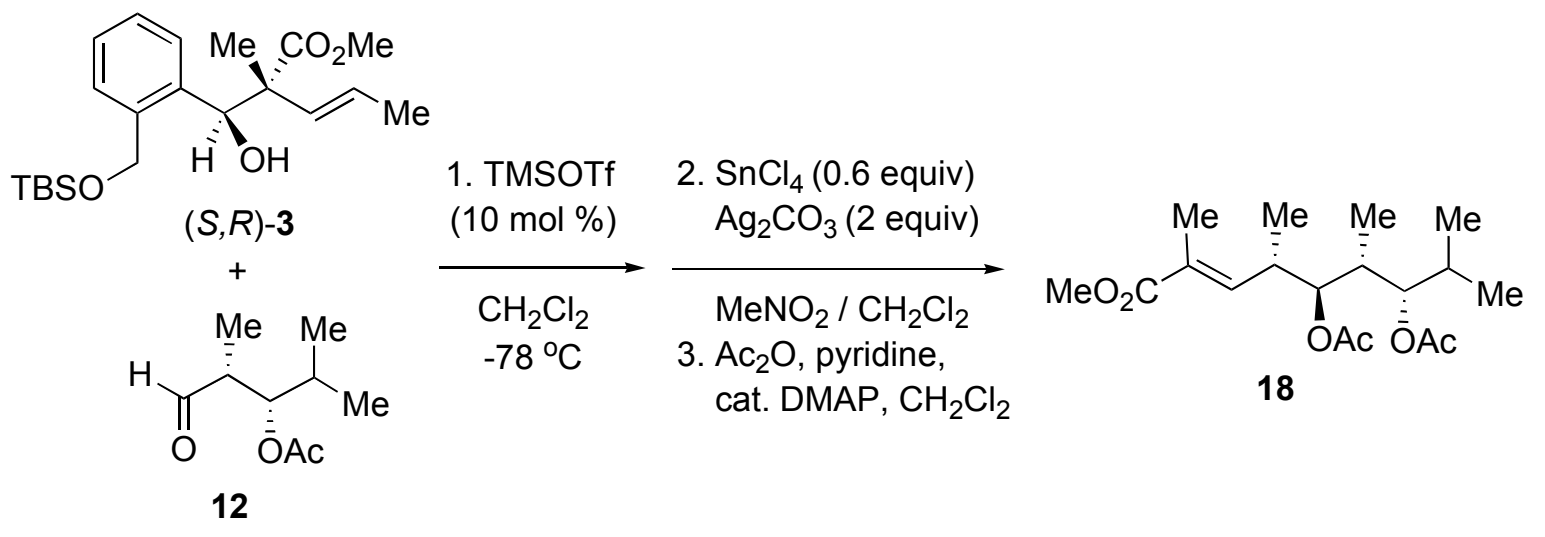

$(4 S, 5 S, 6 R, 7 S)$-5,7-Diacetoxy-2,4,6,8-tetramethylnon-2-enoic acid methyl ester (18). The general procedure B was followed using TMSOTf (2 mg, $0.009 \mathrm{mmol})$, alcohol $(S, R)-\mathbf{3}(35 \mathrm{mg}$, $0.09 \mathrm{mmol}),(2 \mathrm{R}, 3 \mathrm{~S})-3$-acetoxy-2, 4-dimethylpentanal 12 (19 mg, $0.108 \mathrm{mmol}) \mathrm{Ag}_{2} \mathrm{CO}_{3}(50 \mathrm{mg}$, $0.18 \mathrm{mmol})$ and $\mathrm{SnCl}_{4}\left(54 \mu \mathrm{L}, 1 \mathrm{M}\right.$ in $\left.\mathrm{CH}_{2} \mathrm{Cl}_{2}, 0.05 \mathrm{mmol}\right)$. Reaction time: $4 \mathrm{~h}$. The residue was not purified and used for acetylation to provide diacetate 18 in $62 \%$ yield. ${ }^{1} \mathrm{H}$ NMR analysis of the diacetate revealed 9:1 dr. ${ }^{1} \mathrm{H}$ NMR $\left(600 \mathrm{MHz}, \mathrm{CDCl}_{3}\right) \delta 6.68(\mathrm{dq}, 1 \mathrm{H}, J=10.8,1.8 \mathrm{~Hz}), 4.82$ (dd, $1 \mathrm{H}, J=9.6,4.2 \mathrm{~Hz}$ ), 4.78 (dd, 1H, $J=10.2,2.4 \mathrm{~Hz}$ ), 3.75 (s, 3H), 2.95-2.91 (m, 1H), 2.07 (s, $3 \mathrm{H}), 2.02(\mathrm{~s}, 3 \mathrm{H}), 1.94-1.89(\mathrm{~m}, 1 \mathrm{H}), 1.87(\mathrm{~d}, 3 \mathrm{H}, J=1.8 \mathrm{~Hz}), 1.85-1.80(\mathrm{~m}, 1 \mathrm{H}), 0.96(\mathrm{~d}, 3 \mathrm{H}, J=$ $7.2 \mathrm{~Hz}), 0.89$ (d, 3H, $J=6.6 \mathrm{~Hz}), 0.87(\mathrm{~d}, 3 \mathrm{H}, J=7.2 \mathrm{~Hz}), 0.85$ (d, $3 \mathrm{H}, J=6.6 \mathrm{~Hz}) ;{ }^{13} \mathrm{C}(150$ $\left.\mathrm{MHz}, \mathrm{CDCl}_{3}\right) \delta 170.9,170.8,168.5,141.7,128.3,76.3,76.2,51.9,36.0,35.0,30.0,21.1,21.0$, 19.6, 18.8, 16.9, 12.5, 9.8; HRMS (APCI): $\mathrm{m} / z$ calcd. for $\mathrm{C}_{18} \mathrm{H}_{31} \mathrm{O}_{6}\left(\mathrm{M}^{+}\right)$343.2121, found $283.1906\left(\mathrm{M}^{+}\right.$-HOAc); FT-IR (KBr) 2971, 1737, $1238 \mathrm{~cm}^{-1} ;[\alpha]^{25}{ }_{\mathrm{D}}=+25.3\left(\mathrm{c}=0.32, \mathrm{CHCl}_{3}\right)$. 


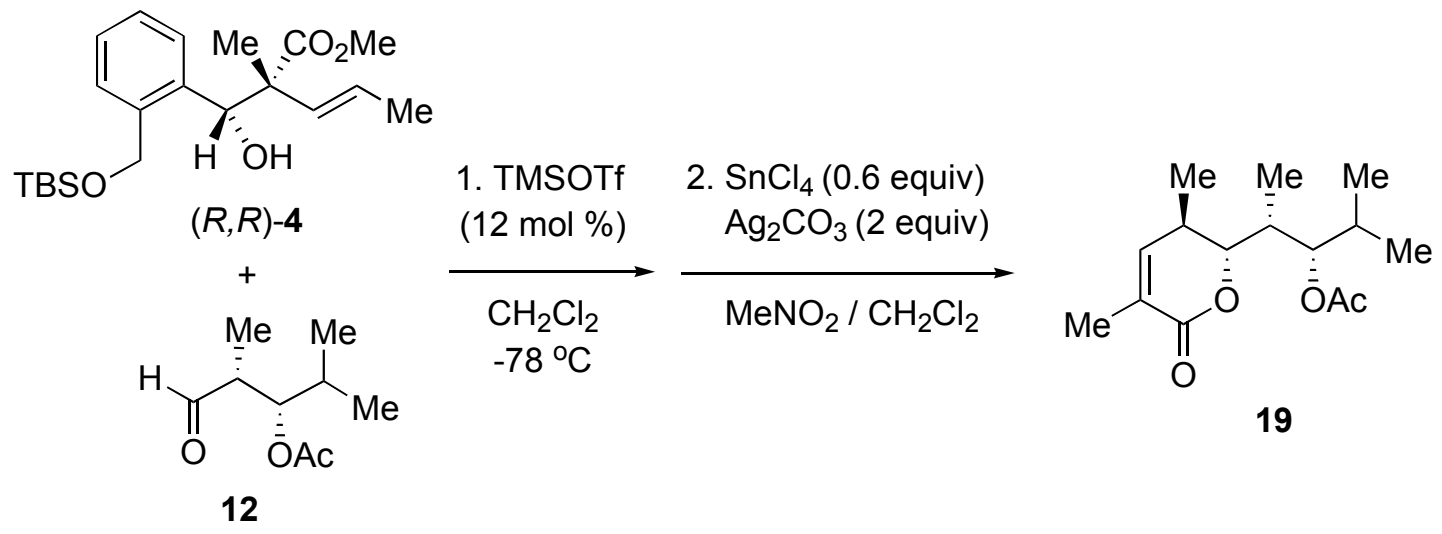

(5R, 6S, 1'R, 2'S)-3,5-Dimethyl-6-(1',3'-dimethyl-2'-acetoxybutyl)-5,6-dihydropyran-2-one (19). The general procedure A was followed using TMSOTf ( $5 \mathrm{mg}, 0.02 \mathrm{mmol})$, alcohol $(R, R)-4$ (62 mg, $0.16 \mathrm{mmol}),(2 R, 3 S)$-3-acetoxy-2, 4-dimethylpentanal 12 (30 mg, $0.17 \mathrm{mmol}) \mathrm{Ag}_{2} \mathrm{CO}_{3}$ (88 mg, $0.32 \mathrm{mmol})$ and $\mathrm{SnCl}_{4}\left(0.1 \mathrm{~mL}, 1 \mathrm{M}\right.$ in $\left.\mathrm{CH}_{2} \mathrm{Cl}_{2}, 0.10 \mathrm{mmol}\right)$. Reaction time: $1.5 \mathrm{~h}$. The residue was purified by flash chromatography on silica gel $($ EtOAc/Hexane $=1: 4)$ to yield $\delta$ lactone 19 (35 mg, 80\%). ${ }^{1} \mathrm{H}$ NMR (400 MHz, $\left.\mathrm{CDCl}_{3}\right) \delta$ 6.33-6.32 (m, $\left.1 \mathrm{H}\right), 4.94(\mathrm{dd}, 1 \mathrm{H}, J=6.4$, $5.6 \mathrm{~Hz}), 4.09$ (dd, 1H, $J=10.4,2.8 \mathrm{~Hz}), 2.66-2.61(\mathrm{~m}, 1 \mathrm{H}), 2.12(\mathrm{~s}, 3 \mathrm{H}), 2.05-1.90(\mathrm{~m}, 2 \mathrm{H}), 1.88$ (dd, $3 \mathrm{H}, J=2.4,1.6 \mathrm{~Hz}$ ), 1.07 (d, $3 \mathrm{H}, J=7.2 \mathrm{~Hz}$ ), 0.99 (d, $3 \mathrm{H}, J=7.2 \mathrm{~Hz}), 0.90$ (d, 3H, $J=6.8$ $\mathrm{Hz}), 0.88(\mathrm{~d}, 3 \mathrm{H}, J=6.8 \mathrm{~Hz}) ;{ }^{13} \mathrm{C}\left(100 \mathrm{MHz}, \mathrm{CDCl}_{3}\right) \delta 171.2,165.2,145.5,127.1,84.6,79.3$, 35.4, 31.0, 28.9, 21.0, 19.4, 17.2, 16.8, 16.4, 8.5 ; FT-IR (KBr) 2968, 1718, $1243 \mathrm{~cm}^{-1}$; HRMS (APCI): $m / z$ calcd. for $\mathrm{C}_{12} \mathrm{H}_{21} \mathrm{O}_{2}\left(\mathrm{M}^{+}\right)$269.1753, found 269.1748; $[\alpha]^{25}=-18.4(\mathrm{c}=0.50$, $\left.\mathrm{CHCl}_{3}\right)$.

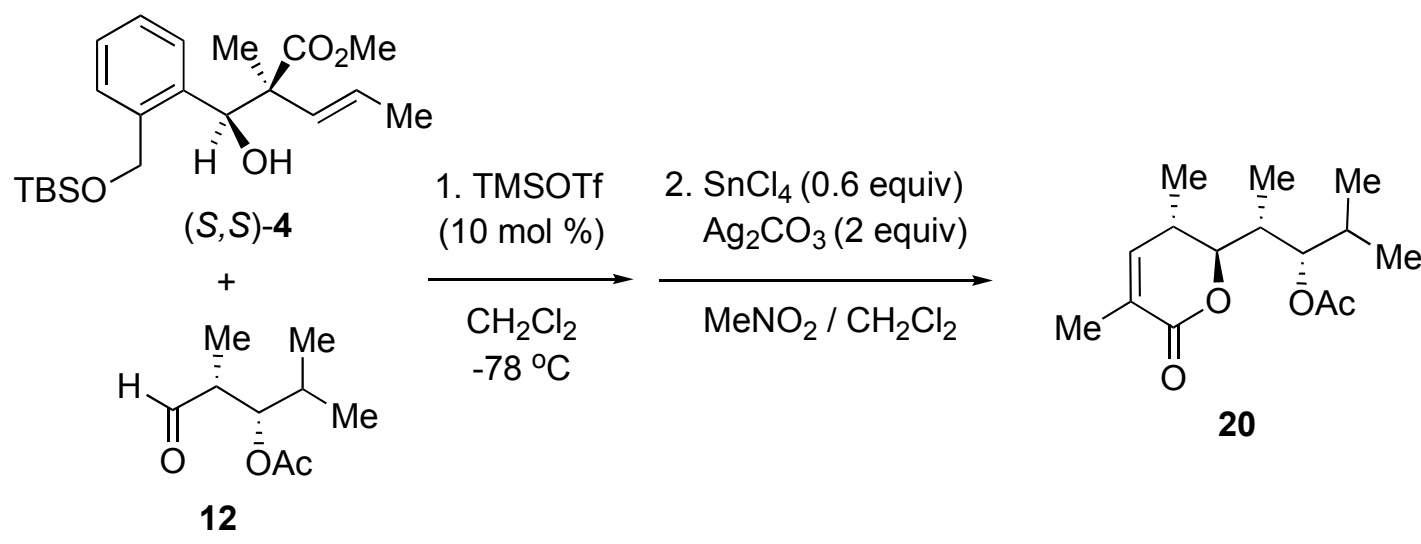

(5S, 6R, 1'R, 2'S)-3,5-Dimethyl-6-(1',3'-dimethyl-2'-acetoxybutyl)-5,6-dihydropyran-2-one (20). The general procedure A was followed using TMSOTf (3 mg, $0.011 \mathrm{mmol})$, alcohol $(S, S)$ 4 (40 mg, $0.11 \mathrm{mmol}),(2 R, 3 S)$-3-acetoxy-2, 4-dimethyl-pentanal (22 mg, $0.13 \mathrm{mmol}), \mathrm{Ag}_{2} \mathrm{CO}_{3}$ 
(61 mg, $0.22 \mathrm{mmol})$ and $\mathrm{SnCl}_{4}\left(0.13 \mathrm{~mL}, 1 \mathrm{M}\right.$ in $\left.\mathrm{CH}_{2} \mathrm{Cl}_{2}, 0.13 \mathrm{mmol}\right)$. Reaction time: $8 \mathrm{~h}$. The residue was purified by flash chromatography on silica gel $(\mathrm{EtOAc} / \mathrm{Hexane}=1: 4)$ to yield $\delta$ lactone 20 (13 mg, 42\%). ${ }^{1} \mathrm{H}$ NMR analysis of the lactone revealed 6:1 dr. ${ }^{1} \mathrm{H}$ NMR (400 MHz, $\left.\mathrm{CDCl}_{3}\right) \delta 6.33-6.32(\mathrm{~m}, 1 \mathrm{H}), 4.90(\mathrm{dd}, 1 \mathrm{H}, J=9.6,1.8 \mathrm{~Hz}$ ), 3.93 (dd, $1 \mathrm{H}, J=10.8,3.6 \mathrm{~Hz}), 2.97-$ $2.94(\mathrm{~m}, 1 \mathrm{H}), 2.11-2.07$ (m, 1H), 2.06 (s, 3H), 1.93-1.89 (m, 1H), 1.87 (d, 3H, J=1.2 Hz), 1.14 $(\mathrm{d}, 3 \mathrm{H}, J=7.2 \mathrm{~Hz}), 1.05$ (d, 3H, $J=7.2 \mathrm{~Hz}), 0.90$ (d, 3H, $J=7.2 \mathrm{~Hz}), 0.89$ (d, $3 \mathrm{H}, J=6.6 \mathrm{~Hz}$ ); ${ }^{13} \mathrm{C}\left(100 \mathrm{MHz}, \mathrm{CDCl}_{3}\right) \delta 171.4,165.4,146.2,126.9,86.8,75.6,34.5,30.5,29.9,21.2,19.0,16.8$, 16.4, 10.9; FT-IR (KBr) 2968, 1722, $1245 \mathrm{~cm}^{-1}$; HRMS (APCI): $\mathrm{m} / z$ calcd. for $\mathrm{C}_{12} \mathrm{H}_{21} \mathrm{O}_{2}\left(\mathrm{M}^{+}\right)$ 269.1753 , found 269.1748 .

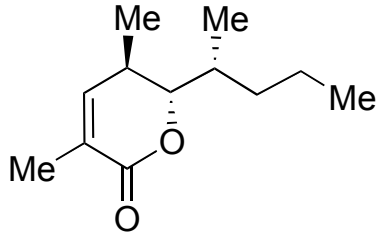

15

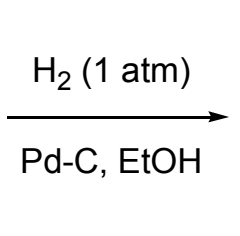

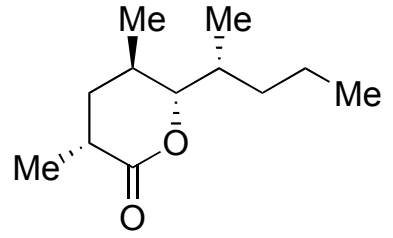

$(-)-21$

(-)-Invictolide (21). A solution of the unsaturated lactone $15(27 \mathrm{mg}, 0.14 \mathrm{mmol})$ and a catalytic amount of $5 \% \mathrm{Pd}$ on carbon $(4 \mathrm{mg})$ in ethanol $(0.3 \mathrm{~mL})$ was stirred at room temperature for $12 \mathrm{~h}$ under one atmosphere of hydrogen (balloon). The reaction mixture was passed through a short plug of silica $($ Ether/Pentane $=1: 10)$ and the solvents were removed by a rotary evaporator to provide a mixture of (-)-invictolide 21 and its 3-epimer (2.33:1) as colorless oil (27 $\mathrm{mg}, 99 \%)$. The ${ }^{1} \mathrm{H}$ - and ${ }^{13} \mathrm{C}$-NMR spectra were fully consistent with those reported by Hoffmann et al. ${ }^{4}$ ${ }^{1} \mathrm{H}$ NMR (400 MHz, $\left.\mathrm{CDCl}_{3}\right) \delta 3.90(\mathrm{dd}, 1 \mathrm{H}, J=9.6,2.0 \mathrm{~Hz}), 2.69-2.59(\mathrm{~m}, 1 \mathrm{H}), 2.02-1.87$ (m, $1 \mathrm{H}), 1.67(\mathrm{t}, 2 \mathrm{H}, J=8.0 \mathrm{~Hz}), 1.51-1.29(\mathrm{~m}, 5 \mathrm{H}), 1.21$ (d, 3H, $J=7.2 \mathrm{~Hz}), 0.97$ (d, 3H, $J=6.8$ $\mathrm{Hz}), 0.91(\mathrm{~d}, 3 \mathrm{H}, J=6.8 \mathrm{~Hz}) ;{ }^{13} \mathrm{C}\left(100 \mathrm{MHz}, \mathrm{CDCl}_{3}\right) \delta 176.8,85.6,36.0,35.3,33.5,32.5,28.3$, 20.4, 17.6, 16.5, 14.1, 12.2; HRMS (APCI): $m / z$ calcd. for $\mathrm{C}_{12} \mathrm{H}_{23} \mathrm{O}_{2}\left(\mathrm{M}^{+}\right)$199.1698, found 199.1693. 


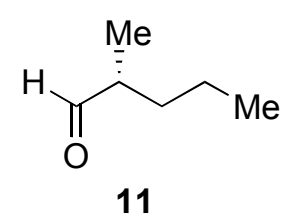

(2R)-2-methylpentanal (11). $n$-BuLi $(4.63 \mathrm{~mL}, 2.5 \mathrm{M}$ in hexane, $11.59 \mathrm{mmol})$ was added to a solution of $\mathrm{LiCl}(1.42 \mathrm{~g}, 33.45 \mathrm{mmol})$ and diisopropylamine $(1.75 \mathrm{~mL}, 12.53 \mathrm{mmol})$ in THF (7 $\mathrm{mL}$ ) at $-78{ }^{\circ} \mathrm{C}$ and the reaction was warmed briefly to $0{ }^{\circ} \mathrm{C}$ and then recooled to $-78{ }^{\circ} \mathrm{C}$. A solution of $(1 R, 2 R)-N$-(2-Hydroxy-1-methyl-2-phenylethyl)- $N$-methylpentanamide ${ }^{5}$ was added dropwise to the reaction flask. The reaction mixture was stirred at $-78^{\circ} \mathrm{C}$ for $1 \mathrm{~h}, 0{ }^{\circ} \mathrm{C}$ for $15 \mathrm{~min}$ and at room temperature for $5 \mathrm{~min}$ and finally cooled to $0{ }^{\circ} \mathrm{C}$ whereupon MeI $(1.39 \mathrm{~mL}, 22.28$ $\mathrm{mmol}$ ) was added. The reaction was stirred at $0{ }^{\circ} \mathrm{C}$ for $55 \mathrm{~min}$ and then quenched by the addition of saturated aqueous ammonium chloride solution. The resulting mixture was extracted with ethyl acetate and the combined organic extracts were dried over sodium sulfate and concentrated to afford yellow oil. Purification of the residue by flash column chromatography on silica gel $($ EtOAc/Hexane $=1: 1)$ to yield amide $(1.38 \mathrm{~g}, 93 \%) .{ }^{1} \mathrm{H}$ NMR $\left(3: 1\right.$ rotamer ratio, ${ }^{*}$ denotes minor rotamer peaks, $\left.400 \mathrm{MHz}, \mathrm{C}_{6} \mathrm{D}_{6}\right) \delta$ 7.35-7.06 (m, 5H), 5.38 (br s, 1H), $4.59(\mathrm{t}, 1 \mathrm{H}, J=6.8$ $\mathrm{Hz}), 4.28 *(\mathrm{dd}, 1 \mathrm{H}, J=8.0,2.4 \mathrm{~Hz}), 4.06-3.96(\mathrm{~m}, 1 \mathrm{H}), 2.87 *$ (s, 3H), 2.83-2.74* (m, 1H), 2.21 (s, 3H), 2.26-2.14 (m, 1H), 1.86-1.70 (m, 2H), 1.35-1.11 (m, 2H), 1.32* (d, 3H, J=6.8 Hz), 1.09 (d, $3 \mathrm{H}, J=7.2 \mathrm{~Hz}), 0.91$ (d, 3H, $J=6.4 \mathrm{~Hz}), 0.83(\mathrm{t}, 3 \mathrm{H}, J=7.2 \mathrm{~Hz}), 0.71 *(\mathrm{~d}, 3 \mathrm{H}, J=6.4 \mathrm{~Hz}$ ); ${ }^{13} \mathrm{C}\left(3: 1\right.$ rotamer ratio, ${ }^{*}$ denotes minor rotamer peaks, $\left.100 \mathrm{MHz}, \mathrm{C}_{6} \mathrm{D}_{6}\right) \delta 178.5,177.5^{*}, 144.3$, $143.3^{*}, 129.0,128.3^{*}, 127.7^{*}, 127.6,127.0,76.8,75.7^{*}, 61.0,58.8^{*}, 37.8^{*}, 36.9,36.8,36.2^{*}$, 34.4, 27.4*, 21.4*, 21.3, 18.7*, 18.1, 16.0* $14.8,14.7$. HRMS (APCI): $m / z$ calcd. for $\mathrm{C}_{16} \mathrm{H}_{26} \mathrm{NO}_{2}$ $\left(\mathrm{M}^{+}\right)$264.1964, found 264.1954; FT-IR (KBr) 3370, 3030, 2961, 1613, $1451 \mathrm{~cm}^{-1}$.

To a solution of diisopropylamine $(1.72 \mathrm{~mL}, 12.31 \mathrm{mmol})$ in THF $(12.5 \mathrm{~mL})$ was added $n$-BuLi $\left(4.76 \mathrm{~mL}, 2.5 \mathrm{M}\right.$ in hexane, $11.43 \mathrm{mmol}$ ) at $-78{ }^{\circ} \mathrm{C}$. The resulting solution was stirred at $-78{ }^{\circ} \mathrm{C}$ for $10 \mathrm{~min}$, then warmed to $0{ }^{\circ} \mathrm{C}$, and held at that temperature for $10 \mathrm{~min}$. Borane-ammonia complex (402 mg, $11.72 \mathrm{mmol}$ ) was added under argon and the suspension was stirred at $0{ }^{\circ} \mathrm{C}$ for $15 \mathrm{~min}$, then warmed to room temperature. After $15 \mathrm{~min}$ at room temperature, the suspension

(4) Hoffmann, R. W.; Ditrich, K.; Köster, G.; Stünner, R. Chem. Ber. 1989, 122, 1783.

(5) Meyers, M. J.; Sun, J.; Carlson, K. E.; Katzenellenbogen, B. S.; Katzenellenbogen, J. A. J. Med. Chem. 1999, 42, 2456. 
was cooled to $0{ }^{\circ} \mathrm{C}$ followed by dropwise addition of a solution of amide $(0.77 \mathrm{~g}, 2.93 \mathrm{mmol})$ in THF $(8.5 \mathrm{~mL})$. Then the reaction was warmed to room temperature and stirred for additional $2 \mathrm{~h}$ before cooling to $0{ }^{\circ} \mathrm{C}$, where excess borane was quenched by careful addition of $3 \mathrm{~N}$ aqueous hydrochloric acid solution $(5 \mathrm{~mL})$. The mixture was stirred for $30 \mathrm{~min}$ at $0{ }^{\circ} \mathrm{C}$ and then extracted with ether. The combined organic extracts were washed sequentially with $3 \mathrm{~N}$ aqueous hydrochloric acid solution $(5 \mathrm{~mL}), 2 \mathrm{~N}$ aqueous sodium hydroxide solution $(5 \mathrm{~mL})$, and brine $(5$ $\mathrm{mL}$ ). The ether extracts were dried over $\mathrm{MgSO}_{4}$ and concentrated. The residue was purified by flash chromatography on silica gel (Ether/Pentane $=1: 2)$ to yield $(2 R)$-2-methylpentanol (219 mg, 73\%). ${ }^{1} \mathrm{H}$ NMR (400 MHz, $\left.\mathrm{CDCl}_{3}\right) \delta$ 3.53-3.40 (m, 2H), 1.67-1.59 (m, 1H), 1.42-1.06 (m, 4H), $0.92-0.89(\mathrm{~m}, 6 \mathrm{H}) ;{ }^{13} \mathrm{C}\left(100 \mathrm{MHz}, \mathrm{CDCl}_{3}\right) \delta 68.4,35.5,35.4,20.0,16.5,14.3 ;[\alpha]_{\mathrm{D}}^{25}=+10.5(\mathrm{c}=$ $0.85, \mathrm{CHCl}_{3}$ ); Mosher ester analysis of the alcohol revealed 98:2 er.

Oxalyl chloride $(0.23 \mathrm{~mL}, 2.56 \mathrm{mmol})$ was dissolved in $\mathrm{CH}_{2} \mathrm{Cl}_{2}(11 \mathrm{~mL})$, and a solution of DMSO $(0.30 \mathrm{~mL}, 4.28 \mathrm{mmol})$ in $\mathrm{CH}_{2} \mathrm{Cl}_{2}(0.75 \mathrm{~mL})$ was added at $-78{ }^{\circ} \mathrm{C}$. The resulting solution was stirred at $-78{ }^{\circ} \mathrm{C}$ for $15 \mathrm{~min}$ followed by dropwise addition of (2R)-2-methyl-1pentanol $(219 \mathrm{mg}, 2.14 \mathrm{mmol})$ in $\mathrm{CH}_{2} \mathrm{Cl}_{2}(2.5 \mathrm{~mL})$. The resulting white heterogeneous mixture was stirred at $-78^{\circ} \mathrm{C}$ for $2 \mathrm{~h}$, and $\mathrm{Et}_{3} \mathrm{~N}(0.90 \mathrm{~mL}, 6.42 \mathrm{mmol})$ was added. After $5 \mathrm{~min}$ at $-78^{\circ} \mathrm{C}$, the mixture was warmed to room temperature for $1.5 \mathrm{~h}$ and $5 \mathrm{~mL}$ of $\mathrm{H}_{2} \mathrm{O}$ was added. The aqueous layer was extracted with $\mathrm{CH}_{2} \mathrm{Cl}_{2}$. The combined organic extracts were washed with brine, dried over $\mathrm{MgSO}_{4}$, and concentrated under reduced pressure. The crude mixture was passed through a short plug of silica $($ Ether/Pentane $=1: 1)$. The aldehyde 11 was used for next step without further purification. ( $150 \mathrm{mg}, 70 \%) .{ }^{1} \mathrm{H} \mathrm{NMR}\left(400 \mathrm{MHz}, \mathrm{CDCl}_{3}\right) \delta 9.62(\mathrm{~d}, 1 \mathrm{H}, J=$ $1.6 \mathrm{~Hz}), 2.38-2.32(\mathrm{~m}, 1 \mathrm{H}), 1.72-1.30(\mathrm{~m}, 4 \mathrm{H}), 1.09$ (d, 3H, $J=7.2 \mathrm{~Hz}), 0.93$ (t, 3H, $J=7.0 \mathrm{~Hz})$; ${ }^{13} \mathrm{C}\left(100 \mathrm{MHz}, \mathrm{CDCl}_{3}\right) \delta 205.5,46.1,32.6,20.1,14.0,13.2$; FT-IR (KBr) 2864, 2715, 1710, $1388 \mathrm{~cm}^{-1} ;[\alpha]^{25}{ }_{\mathrm{D}}=-27.4(\mathrm{c}=0.42$, acetone $)$. 


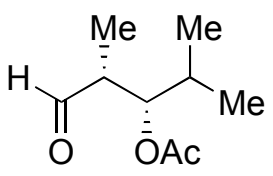

12

(2R, 3S)-3-Acetoxy-2,4-dimethylpentanal (12). To a solution of (3S, 4S)-2,4-dimethyl-3hydroxy-5-hexene ${ }^{6}(519 \mathrm{mg}, 4.05 \mathrm{mmol})$ in $\mathrm{CH}_{2} \mathrm{Cl}_{2}(12 \mathrm{~mL})$ was added pyridine $(0.98 \mathrm{~mL}$, $12.15 \mathrm{mmol})$, acetic anhydride $(0.76 \mathrm{~mL}, 8.10 \mathrm{mmol})$ and DMAP $(25 \mathrm{mg}, 0.20 \mathrm{mmol})$ at $0{ }^{\circ} \mathrm{C}$. The resulting solution was allowed to warm to room temperature and stirred for another $2 \mathrm{~h}$ before quenched with water. The extractions was performed with $\mathrm{CH}_{2} \mathrm{Cl}_{2}$ and the combined organic layers were dried $\left(\mathrm{MgSO}_{4}\right)$, filtered, and concentrated. The residue was purified by flash chromatography on silica gel $($ EtOAc/Hexane $=1: 8)$ to yield acetate $(681 \mathrm{mg}, 98 \%) .{ }^{1} \mathrm{H}$ NMR

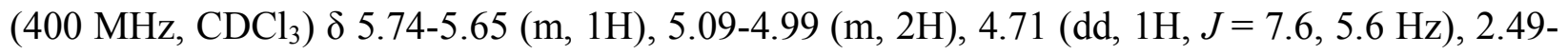
$2.43(\mathrm{~m}, 1 \mathrm{H}), 2.07(\mathrm{~s}, 3 \mathrm{H}), 1.94-1.86(\mathrm{~m}, 1 \mathrm{H}), 0.97(\mathrm{~d}, 3 \mathrm{H}, J=6.8 \mathrm{~Hz}), 0.88(\mathrm{~d}, 3 \mathrm{H}, J=3.8 \mathrm{~Hz})$, $0.87(\mathrm{~d}, 3 \mathrm{H}, J=3.8 \mathrm{~Hz}) ;{ }^{13} \mathrm{C}\left(100 \mathrm{MHz}, \mathrm{CDCl}_{3}\right) \delta 171.1,140.5,114.7,80.4,39.7,29.5,20.9$, 19.7, 16.5, 15.2; FT-IR (KBr) 2972, 1741, $1239 \mathrm{~cm}^{-1}$; HRMS (APCI): $\mathrm{m} / z$ calcd. for $\mathrm{C}_{10} \mathrm{H}_{19} \mathrm{O}_{2}$ $\left(\mathrm{M}^{+}\right)$171.1385, found 171.1380; $[\alpha]^{25}=-17.8\left(\mathrm{c}=0.84, \mathrm{CHCl}_{3}\right)$.

A solution of (3S, 4S)-2,4-dimethyl-3-acetoxy-5-hexene (576 mg, $3.34 \mathrm{mmol})$ in $1: 1 \mathrm{CH}_{2} \mathrm{Cl}_{2}$ : $\mathrm{MeOH}(24 \mathrm{~mL}, 0.08 \mathrm{M})$ was added pyridine $(0.16 \mathrm{~mL}, 2 \mathrm{mmol})$ at room temperature under argon. The resulting solution was cooled to $-78{ }^{\circ} \mathrm{C}$ and bubbled into $\mathrm{O}_{3}$ until starting material was consumed. $\mathrm{Me}_{2} \mathrm{~S}(5 \mathrm{~mL}, 60 \mathrm{mmol})$ was added to the flask and the reaction was allowed to warm slowly to room temperature over a period of $18 \mathrm{~h}$. The crude mixture was concentrated and diluted with ethyl acetate $(40 \mathrm{~mL})$. The organic layer was washed with water and brine followed by drying over $\mathrm{MgSO}_{4}$, filtered and concentrated. The crude aldehyde 12 (495 mg, 86\%) was used in the next transformation. further purification. ${ }^{1} \mathrm{H} \mathrm{NMR}\left(400 \mathrm{MHz}, \mathrm{CDCl}_{3}\right) \delta 9.67(\mathrm{~d}, 1 \mathrm{H}$, $J=1.2 \mathrm{~Hz}), 5.14(\mathrm{dd}, 1 \mathrm{H}, J=8.0,4.0 \mathrm{~Hz}), 2.70-2.64(\mathrm{~m}, 1 \mathrm{H}), 2.04(\mathrm{~s}, 3 \mathrm{H}), 1.96-1.90(\mathrm{~m}, 1 \mathrm{H})$, $1.08(\mathrm{~d}, 3 \mathrm{H}, J=6.8 \mathrm{~Hz}), 0.95(\mathrm{~d}, 3 \mathrm{H}, J=6.8 \mathrm{~Hz}), 0.93(\mathrm{~d}, 3 \mathrm{H}, J=6.8 \mathrm{~Hz}) ;{ }^{13} \mathrm{C}(100 \mathrm{MHz}$, $\left.\mathrm{CDCl}_{3}\right) \delta 202.3,170.7,76.3,48.6,29.8,20.7,19.0,18.3$, 7.4; FT-IR (KBr) 2972, 2880, 1733 , $1374 \mathrm{~cm}^{-1}$; HRMS (APCI): $m / z$ calcd. for $\mathrm{C}_{10} \mathrm{H}_{19} \mathrm{O}_{2}\left(\mathrm{M}^{+}\right)$173.1178, found 173.1173 .

(6) (a) Burova, S. A.; McDonald, F. E.; J. Am. Chem. Soc. 2004, 126, 2495. (b) Dreher, S. D.; Leighton, J. L. J. Am. Chem. Soc. 2001, 123, 341. This compound was produced in $85 \%$ ee (determined by Mosher ester analysis). 